\title{
A data multiverse analysis investigating non-model based SCR quantification approaches
}

\section{Short title: multiverse analyses on SCR quantification}

\author{
Rachel Sjouwerman ${ }^{1}$, Sabrina Illius ${ }^{1}$, Manuel Kuhn ${ }^{1,2}$ \& Tina B. Lonsdorf ${ }^{1}$ \\ ${ }^{1}$ Institute for Systems Neuroscience, University Medical Center Hamburg-Eppendorf, Hamburg, Germany \\ ${ }^{2}$ Department of Psychiatry, Harvard Medical School, and Center for Depression, Anxiety and Stress \\ Research, McLean Hospital, Belmont, MA 02478 USA.
}

Impact statement: This study navigates through the robustness of different non-model based skin conductance quantification approaches. Systematic multiverse perspectives on different ways to quantify the data highlight that even with overall high agreement and replicability of large effects between different quantification approaches, caution is warranted in light of systematic biases.

Keywords: baseline correction, trough-to-peak, SCR, SCL, specification curve, multiverse 


\begin{abstract}
Electrodermal signals are commonly used outcome measures in research on arousal, emotion, and habituation. Recently, we have reported on heterogeneity in skin conductance response quantification approaches and its impact on replicability. Here we provide complementary work focusing on withinapproach heterogeneity of specifications for skin conductance response quantification. We focus on heterogeneity within the baseline-correction approach (BLC) which appeared as particularly heterogeneous - for instance with respect to the duration of the pre-CS baseline window, the start and end of the peak detection window. Here we systematically scrutinize the robustness of results when applying different BLC approach specifications to one pre-existing dataset $(\mathrm{N}=118)$ in a pre-registered study. We report high agreement between different BLC approaches for US and CS+ trials, but moderate to poor agreement for CS- trials. Furthermore, a specification curve of the main effect of CS discrimination during fear acquisition training from all potential and reasonable combinations of specifications $(\mathrm{N}=150)$ and a prototypical trough-to-peak (TTP) approach indicates that resulting effect sizes are largely comparable. Crucially, however, we show that BLC approaches often misclassify the peak value particularly for CS- trials, which leads to a stimulus-specific bias and challenges for post-processing and replicability. Lastly, we investigate how physiologically implausible (negative) skin conductance values in BLC, appearing most frequently for CS- (CS- > CS+ > US), correspond to values in TTP quantification. We discuss the results in terms of robustness and replicability and provide insights into challenges, opportunities, and implications.
\end{abstract}




\section{Introduction}

Skin conductance responding is a peripheral physiological measure that is used to infer sympathetic nervous system activation and is commonly used in studies on emotion, attention, habituation and arousal (Dawson et al., 2007). Yet, heterogeneous approaches to quantify skin conductance responses (SCRs) coexist and can make it difficult to compare results across studies and raise questions on replicability. Recently, we (Kuhn et al., 2021) systematically compared the replicability of skin conductance responding between different categories of skin conductance response quantification approaches by applying seven different approaches to two different sets of raw data. Using fear conditioning research as a case example, we concluded that results do not necessarily replicate between response quantification categories and that no single approach consistently produced larger effect sizes across different paradigms. More precisely, group-level trial-by-trial agreement across different approaches was fair to substantial with some evidence for stimulus-type dependent agreement rates (Kuhn et al., 2021). Of note, the systematic literature review included in this previous work (Kuhn et al., 2021) did not only reveal that different categories of approaches co-exist but also brought the substantial methodological heterogeneity within individual SCR quantification categories to our attention. This within-approach heterogeneity represents an additional challenge to the comparability and interpretation of study results as studies that use the same approach do not necessarily converge in methods. Here, we focus on within-approach heterogeneity in the so-called 'baseline-correction approach' (BLC) category which appeared as particularly heterogeneous with respect to specifications used to identify a SCR in our literature search.

\subsection{Brief introduction into skin conductance and response quantification}

The skin conductance signal comprises a slowly changing tonic component (skin conductance level, $\mathrm{SCL}$ ) and a faster changing phasic component characterized by a sharp inflection (i.e., steep incline) in the signal and its subsequent return (skin conductance response, SCR). SCRs occur both spontaneously (i.e., nonspecific fluctuations) and stimulus-bound (Boucsein et al., 2012) with the latter being the focus of our previous (Kuhn et al., 2021) and present work. In psychophysiological research, both SCR and SCL are extensively used (Boucsein et al., 2012) and will be discussed in this work with a focus on SCRs.

As mentioned above, SCRs can be quantified though different approaches including model-based approaches as implemented in the software-packages Ledalab (Benedek \& Kaernbach, 2010; Lim et al., 1997) or PsPM (Bach et al., 2009, 2013; Bach \& Friston, 2013) and non-model-based approaches such as computer-assisted or (semi-)manual Trough-to-peak (TTP) scoring and baseline correction approaches (BLC). We refer the interested reader to our previous work (Kuhn et al., 2021) for a detailed discussion and comparison of these different approaches. As such, the present work provides a complementary extension of Kuhn et al., (2021) by using the same set of raw data to investigate within-approach heterogeneity in the BLC approach and by using the TTP approach (as also implemented in Kuhn et al., 2021) as a reference approach.

\subsubsection{SCR quantification in non-model based approaches}

Both BLC and TTP approaches are subsumed under the term 'non-model based SCR quantification approaches'. In TTP scoring, the SCR amplitude is defined as the difference between the skin conductance at the peak of the response and its preceding trough. The time-windows during which the peak and trough

are identified are defined according to a published set of empirically-derived criteria (Boucsein, 2012): The trough (i.e., footpoint of the SCR) is typically required to occur with a latency of 1-3s (Levinson \& Edelberg, 1985), 1-3.5s (although stimulus type-specific response windows were suggested; Sjouwerman \& Lonsdorf, 2019), or 1-4s (Boucsein et al., 2012) after stimulus onset. The peak value of the SCR is typically 
required to occur in a time window of 0.5-5s after the onset of the SCR (Boucsein et al., 2012; Grings, 1974). Here we refer to this as the peak detection window (PDW). For example, if the trough of the SCR occurs $2 \mathrm{~s}$ after the stimulus presentation, then the peak must occur in a PDW of 2.5-7s after stimulus onset. In addition, a minimum response amplitude is often applied that typically varies between $0.01 \mu \mathrm{S}$ to $0.05 \mu \mathrm{S}$ (Boucsein et al., 2012; Lonsdorf, Klingelhöfer-Jens, et al., 2019). SCR amplitudes smaller than this minimum response amplitude are typically considered as non-responses with a value of zero (Venables, P.H. \& Christie, M.J., 1980). In fear conditioning research, which we use as a case example here, a distinction has been suggested between first, second, and third interval responses with different response onset time windows (OTW's) provided a sufficiently long stimulus presentation is given (Stewart et al., 1961, or an overview of used OTW's see Pineles et al., 2009). Although not universally agreed upon, these three intervals have been suggested to capture different processes (for a summary and critique see Pineles et al., 2009).

The BLC approach originates from a critical evaluation of the difference between the first and second interval response in differential fear conditioning studies and an attempt to provide a tool that neither requires mathematical modelling nor learning new software or user-specific decisions. This led to the recommendation of an 'entire-interval response' in which the highest skin conductance value (i.e., peak) during the entire stimulus presentation time is selected (Pineles et al., 2009). Quantifying the EIR was originally based on an algorithm in the software program Mathematica that identified the onset of the SCR in the entire stimulus presentation window which served also as the PDW. The response peak was then identified within the PDW as the highest value after the identified onset (Pineles et al., 2009). Accordingly, the entire interval response was calculated by subtracting the mean skin conductance level averaged over the $2 \mathrm{~s}$ immediately preceding stimulus onset (i.e., baseline window, BLW) from the highest skin conductance value during the entire stimulus presentation period (i.e., a PDW and CS duration of 8s in Pineles et al., 2009). Note that, while the PDW in this BLC approach corresponds to the full stimulus duration, in TTP, the PDW starts at the onset of the SCR, which per definition is later than the stimulus onset due to SCR occurring with a latency of 1-4s (see above).

More precisely and importantly, the algorithm suggested by Pineles required that neither the onset of the SCR nor its peak may be located at the first or last data point of their respective time windows to avoid selecting non-stimulus bound responses. If this was the case for a given trial, the algorithm rejected the onset and peak and looked for a new onset and peak in a shrunken window. If the onset and peak were rejected, the window was iteratively shrunken and no response identified when it reached a zero-width. This approach was subsequently adopted and implemented in custom-made scripts which identify a peak in a PDW and subtract an average value during a baseline window (BLW) - however, and importantly (mostly) without the iterative algorithm as described above. Of note and in contrast to TTP scoring, this procedure can yield negative magnitudes for a SCR when no stimulus-bound response is observed or when it is comparably smaller than the (habituation) drift. Some, but not all authors set these negative responses explicitly to "zero" during post-processing (Mertens et al., 2019; Vogel et al., 2015). Notably, baselinecorrection approaches are also applied for the quantification of other physiological measures than SCR, such as EEG (Alday, 2019) and pupillometry (Mathôt et al., 2018).

\subsubsection{Investigating different specifications of SCR quantification in BLC approaches - a data multiverse approach}

As pointed out, our previous and related work (Kuhn et al., 2021) has revealed substantial heterogeneity in specification of the criteria used to define an SCR in the BLC approach. Yet, we did not systematically carve out this heterogeneity (e.g., start and end of the PDW, BLW duration, mean or maximum value and 
their combination) nor did we investigate its impact on the results - both of which is the focus of the present work.

A promising approach to systematically and comprehensively explore the impact of heterogeneous (though equally justified) specifications on an effect of interest are multiverse-style analyses such as the 'multiverse analysis' (Steegen et al., 2016), the related 'specification curve analysis' (Simonsohn et al., 2020) or the vibration of effects (Patel et al., 2015). Multiverse-style analyses depart from the fact that there is a (large) number of decisions a researcher has to make during the scientific process from data processing to analysis and interpretation. For instance, a single set of raw data can be processed into a multiverse of different processed datasets (the 'multiverse'), whereof in a typical study only a single processed dataset is generated from the raw data (a 'universe', Steegen et al., 2016) by making specific choices at each decision nod. The different decisions are often equally justifiable in absence of empirical and/or theoretical criteria to guide the researchers' decisions. Similar to the above described 'data multiverse', many equally justifiable statistical models can be applied to the data to answer the same hypothesis which is referred to as the 'model multiverse' (Steegen et al., 2016). Of note, also the data multiverse inevitably implies a multiverse of statistical results given that the same statistical models are applied to differently processed data that however are derived from identical raw data. This has been highlighted for psychology in general (Silberzahn et al., 2018; Steegen et al., 2016) and for fear conditioning research in particular (Lonsdorf, Klingelhöfer-Jens, et al., 2019; Lonsdorf, Merz, et al., 2019, Lonsdorf et al., 2021). To this end, multiverse-style data analyses can be considered a large set of systematic robustness analyses that apply different (equally justifiable) processing pipelines to the same dataset. In fact, such analyses with one dataset have the advantage that they reduce error variance and deviations will derive from processing rather than from sample differences. It is, however, important to point out that the multiverse can be "a dangerous place" (DelGuidice \& Gangestad, 2021) as the inclusion of nonequivalent decisions may lead to a 'combinatorial explosion' which can cause true effects to be swallowed by 'analytical black holes'. We refer to the excellent discussion by DelGuidice \& Gangestad (2021) on the potentials and pitfalls of multiverse-style analyses.

Here, we present a data-multiverse analysis focusing on different SCR quantification specifications using the BLC method and one prototypical TTP approach as a reference. As the definition of the different specifications to be included in multiverse-type of analyses is central to the information the study can provide (DelGuidice et al., 2021), we derive the specifications used to generate the multiverse from a systematic literature search based on our related work (Kuhn et al., 2021) which used fear conditioning research as a case example.

First, we report more detailed results from a systematic literature search (see Kuhn et al., 2021) providing information on which parameters and specifications are used for BLC-based SCR quantification in the field of fear conditioning research. Second, we conduct a small multiverse-type of analysis in which we apply nine literature-derived unique BLC specifications, to a single dataset. We provide information on the effect of interest in fear conditioning studies (i.e., CS discrimination), the effect sizes across these nine specifications, and compare them to the results derived from a prototypical TTP approach. Third, we generate a full multiverse by employing all (meaningful and in principle equally justifiable) potential combinations of the specific decision nods as identified by the systematic literature search to systematically delineate the impact of any individual specification. Results are presented in the form of a specification curve (Simonsohn et al., 2020) and can be expected to provide insights into the robustness of SCRs and the effect of interest against methodological specifications and further our understanding of replicability. 
Finally, we elaborate on the question of how to interpret negative magnitudes derived from the BLC approach in relation to the prototypical TTP approach. 


\section{Method}

\subsection{Systematic literature search}

A systematic literature search covering six months (22.9.2018 - 22.3.2019) was conducted according to PRISMA guidelines (Moher et al., 2009). The search covered all publications and e-pubs ahead of print listed in PubMed for this time window and was reported in detail in our previous work focusing on a different topic (Lonsdorf, Klingelhöfer-Jens, et al., 2019) and also mentioned in our previous, related work (Kuhn et al., 2021). In brief, 854 records listed in PubMed were identified for which 152 records were retained for stage 2 screening (abstract). Only 86 records were retained for stage 3 screening (full text) and the final set of studies consisted of 50 records that reported results for (1) skin conductance as an outcome measure from (2) the fear acquisition training phase (3) in human participants (a flow chart with details has is included in (Lonsdorf, Klingelhöfer-Jens, et al., 2019)). For each record, we extracted information on how skin conductance responses were quantified with the aim to apply the different approaches as identified from the literature to our own data. These results were also relevant to our related work, which focused on a direct comparison between seven different SCR response quantification approaches (TTP, BLC, Ledalab, PsPM, Kuhn et al., 2021) while we focus on within-approach heterogeneity here.

\subsection{Pre-registration}

This project has been pre-registered on the Open Science Framework (OSF) (June 24, 2020; retrieved from https://osf.io/rfy6q/). Deviations from the pre-registered protocol are made explicit in this manuscript and the reasons for deviations are specified. Originally the focus was only on applying the exact specifications from the literature to our pre-existing dataset. Here we go one step further and include an additional notpreregistered full multiverse to allow for an even more systematic evaluation of the potential impact of variations in BLC specifications.

The pre-registered hypothesis regarding individual differences in fear conditioning is omitted as it would exceed the scope of the current manuscript.

\subsection{Participants}

One-hundred and twenty participants were included in a longitudinal fear conditioning study for which we only analyze data from the fear acquisition training phase at the first assessment time point. Data from two participants were excluded due to protocol deviations leaving 118 participants for analyses (78 females, mean \pm SD age of $24.4 \pm 3.7$ years). Written informed consent was obtained from all participants and the project was approved by the local ethics committee (PV 5157, Ethics Committee of the General Medical Council Hamburg). Data analyzed here have been included as a case example on a different methodological question (i.e., exclusion of 'non-learners' and 'non-responders') in our previous publication (Lonsdorf, Klingelhöfer-Jens, et al., 2019), our recent related work on a direct comparison of different SCR response quantification approaches (Kuhn et al., 2021) as well as an exemplary data set for the development of a methods multiverse package for the software package R (Lonsdorf et al., 2021).

\subsection{Paradigm and Stimuli}

The paradigm has been described before in detail (for details see Lonsdorf, Klingelhöfer-Jens, et al., 2019) and the study included a baseline measurement (TO) and a follow-up measurement (T1) six month later. An identical paradigm was conducted at both timepoints. In brief, it consisted of a two-day uninstructed fear conditioning paradigm with habituation and acquisition training on day 1 and extinction training and recall test on day 2. BOLD fMRI, fear ratings (after each experimental phase), and skin conductance 
responses were acquired during all experimental phases but only results for SCRs during fear acquisition training at T0 are reported here as the focus of the present work is on a methodological question of SCR response quantification.

Conditioned stimuli were two light grey fractals presented 14 times each in a pseudo-randomized order for 6-8 s (mean: $7 \mathrm{~s}$ ). The CS+ was always followed by the US during fear acquisition training (100\% reinforcement rate) and allocation of CS+ and CS- to the stimuli was counterbalanced. A white fixation cross served as the inter-trial intervals (ITIs) and was presented for 10-16 s (mean: $13 \mathrm{~s}$ ). All stimuli were presented on a grey background and experimental timing was controlled by Presentation software (Version 14.8, Neurobehavioral Systems, Inc, Albany California, USA).

The US was administered to the back of the right hand and consisted of an electrotactile stimulus (three $2 \mathrm{~ms}$ electrotactile rectangular pulses with an interpulse interval of $50 \mathrm{~ms}$ ) that started $.2 \mathrm{~s}$ before CS+ offset. The US was generated by a Digitimer DS7A constant current stimulator (Welwyn Garden City, Hertfordshire, UK) and administered through a $1 \mathrm{~cm}$ diameter platinum pin surface electrode (Speciality Developments, Bexley, UK) attached between the metacarpal bones of the index and middle finger. The intensity of the US intensity was individually calibrated in a standardized step-wise procedure to generate an intensity that was described as unpleasant, but still tolerable.

\subsection{Skin conductance recording}

Skin conductance response was measured via self-adhesive $\mathrm{Ag} / \mathrm{AgCl}$ electrodes placed on the palmar side of the left hand on the distal and proximal hypothenar. Data was recorded at $1000 \mathrm{~Hz}$ with a Biopac MP150amplifier system (BIOPAC ${ }^{\circledR}$ Systems Inc., Goleta, CA, USA) including a skin conductance unit and converted from analog to digital using a CED2502-SA and the Spike 2 software (Cambridge Electronic Design, Cambridge, UK). Physiological data were recorded continuously at $1000 \mathrm{~Hz}$ with a gain of $5 \mu \Omega$.

Note, that in the pre-registration erroneously specified the Biopac MP100 amplifier (BIOPAC Systems Inc. Goleta, California, USA) and AcqKnowledge 3.9.2 software. Furthermore, we used untransformed skin conductance values rather than log-transformed and range-corrected values. This deviates from the preregistered protocol because we considered it more informative for the purpose here to compare the untransformed values unaffected by the highest response (used for range-correction).

\subsection{SC response quantification}

Skin conductance responses (SCRs) were quantified through a number of different approaches as identified through the systematic literature search (see results for details). In this report, we focus on the 'baseline correction' category and its different specifications in comparison to one prototypical approach for trough-to-peak scoring. In the pre-registration, we stated that we include data from an experienced and a first-time TTP rater. Deviating from this pre-registered plan, we only include data derived from the experienced TTP rater as a direct comparison of inter-rater reliability was a better fit for a different manuscript and is hence reported there (Kuhn et al., 2021).

\subsubsection{Trough-to-peak (TTP)}

Data were down-sampled to $10 \mathrm{~Hz}$ and responses were quantified offline while being blind to stimulus type using a custom-made computer program and according to published guidelines (Boucsein et al., 2012). More precisely, the trough was identified in an onset latency window (OLW) of 0.9 to 4 s poststimulus onset and the peak was identified in a peak detection window (PDW) after response onset until maximally $5 \mathrm{~s}$ post-SCRonset. If multiple peaks were identified in the PDW, the first peak was considered. 
Each response identified by the custom-made program was checked visually, and adjusted manually if necessary (e.g., the foot or trough was misclassified by the algorithm). Data were treated as missing in case of recording artifacts or excessive baseline activity (i.e., more than half of the response amplitudes) and thus omitted from analyses. Responses below a minimal amplitude of $0.01 \mu \mathrm{S}$ or the absence of any response (i.e., flat line or habituation drift) within the defined time window were classified as nonresponses (i.e., zero) and included in the analyses. The $0.01 \mu \mathrm{S}$ threshold was determined empirically by visually inspecting responses specifically above and below this cutoff as reported previously for this dataset (Lonsdorf, Klingelhöfer-Jens, et al., 2019). Data and results from the TTP approach as employed here are identical with data and results of the TTP approach (rater 2) as reported in our previous work (Kuhn et al., 2021). Here, the TTP approach served as a reference approach while in our previous and complementary work (Kuhn et al., 2021), the TTP approach was directly compared to the TTP approach employed by a different rater (TTP, rater 1, a prototypical BLC approach (corresponding to BLC7 here with a BLW of $2 s$ and PDW of $6 s$ ) as well as to different model-based approaches as implemented in the software packages Ledalab and PsPM.

\subsubsection{Baseline correction (BLC)}

A custom-made script in Matlab, version R2018a implemented the BLC response quantification approach by subtracting the mean of pre-CS onset window (baseline window, BLW) from the subsequent maximum identified in a peak detection window (PDW) for SCR or the average during the PDW for SCL. Inbuilt Matlab functions mean() and $\max ()$ are used to select respective values in to be defined time windows. Nine different specifications of the BLW, the PDW as well as the maximum or the average during the PDW (aggregate function) as derived from the systematic literature search were implemented which are described in detail in the results (see section 3.1). Formulas reported in the literature that a priori do not make sense when applied to the experimental design of our exemplary dataset are excluded. More extensive multiverse analyses with BLCs are described in section 2.9.

\subsection{Statistical evaluation of Replication outcome}

We refrain from the pre-registered ANOVAs with mean and trial-by-trial SCR values to investigate differences of the effect of SCR quantification approaches as comparing $x$ different BLC approach groups seemed after reconsideration infeasible and uninformative on a trial-by-trial level considering the number of post hoc tests and redundant for the mean effect when also including a specification curve. Instead, as in our related work (Kuhn et al., 2021) we implemented a framework to evaluate replication of main effects of average CS responding across different approaches in a full data multiverse (section 2.7 and 2.9), as well as agreement analyses between approaches based on Krippendorff's $\alpha$ (section 2.8) on average as well as trial-by-trial levels.

Here, we employ a replication framework to quantify whether the different specifications of the BLC approach for SCR quantification yield comparable results when applied to an identical dataset (i.e., robustness analyses). This evaluation was not pre-registered. More precisely, we aim to empirically evaluate whether these different specifications of BLC approaches as well as the prototypical TTP approach can be considered exact/very close replications of each other or have to be considered far (or conceptual) replications in the data-set used here. To this end, we adopt the framework suggested by LeBel and colleagues (LeBel et al., 2018) for the evaluation of replication attempts and apply it to the robustness analyses in this work (i.e., applying different methods to identical data. This extension also includes further steps (see section 2.2) that go beyond the descriptive assessment of agreement across SC quantification 
approaches stated in the pre-registration. More precisely, according to LeBel's suggestion, we evaluate the following criteria (note, however, that LeBel used an NHST framework, while we use a Bayesian approach here):

a) Signal detection (yes/no). A signal is considered detected within an approach when the $95 \% \mathrm{Crl}$ around the effect size point estimate excludes zero.

b) The precision of the estimated effect size. How wide are the Crl's within the different SCR quantification approaches?

c) The consistency of the estimated effect sizes. Consistency between two effects is considered given when the effect size point estimate of one SCR quantification approach is included in the other effect size's Crl's.

\subsection{Measures of agreement across SCR quantification approaches}

Krippendorff's $\alpha$ will be used as a measure of reliability and reflects the (dis)agreement between approaches. This measure is optimally suited to compare more than two categories of quantification approaches, it can handle missing data, and can be applied to any measurement scale (Zapf et al., 2016). Here, we will use an ordinal scale of skin conductance values to establish an index of rank stability rather than absolute numerical agreement. Krippendorff's $\alpha$ is a reliability coefficient with values ranging from 1 to 1 , where -1 reflects perfect disagreement, 0 no agreement beyond chance, and 1 is indicative of perfect agreement. According to Krippendorff, an $\alpha$ of $\geq .8$ is required for agreement (Krippendorff, 2004). Benchmark values have been suggested (Landis et al., 1977) for interpretation of the strength of agreement as almost perfect (1-.81), substantial (.8-.61), moderate (.6-.41), and fair (.40-.21). Note that these divisions are arbitrary but provide useful benchmarks and should hence be interpreted as such. Krippendorff's $\alpha$ will be calculated using the R syntax available from additional file 4 in Zapf et al. (2016), with bootstrap confidence intervals $(n=1000)$. This analysis was not pre-registered, but was added as it provides helpful guidance in understanding convergence between approaches.

\subsection{Full data multiverse}

The subsequent analysis was not pre-registered, but is included to allow for a systematic investigation of the effects of different specifications of the BLC approach (i.e., within-approach heterogeneity) on skin conductance quantification and to enhance understanding of the relationship between BLC values and TTP values.

To investigate the impact of the variation in the four identified parameters (i.e., BLW, PDW-start, PDWend, aggregate function, see Figure 1) of the BLC formulas as reported in the literature more systematically, a full data multiverse was generated including, all $n$ possible unique combinations of specifications found in the published studies. The PDW specification was operationalized relative to a fixed CS duration (i.e., $6 \mathrm{~s}$ which is the minimum CS duration in the current dataset). For example a PDW-start at $1 \mathrm{~s}$ and PDW-end at $5 \mathrm{~s}$ as derived from the literature was translated here by using our fixed CS duration of $6 \mathrm{~s}$. This results in the following formula CS on $+1 \mathrm{~s}$ and CS off $-1 \mathrm{~s}$ (i.e., 1-5s). Here we applied all these $n$ combinations of BLC specifications to the unprocessed continuously recorded skin conductance signal and generated a multiverse of $\mathrm{n}$ different ways of quantifying SCRs based on all possible combinations of the criteria identified for SCR quantification through BLC. 
This was done with a custom-made script (available at https://github.com/rsjouwerman/repl-ttp-blc) that applied these criteria to each individual trial during fear acquisition training (ie., 14 trials of each CS+, CSand US) resulting in a total of 42 (trials) $\mathrm{x} n$ (approaches) single trial SCRs computed for each of the 118 participants (i.e., in total $118 \times 42 \times \mathrm{n}$ values). Importantly, the full data multiverse includes combinations of specifications that were not originally identified in the literature. One combination of specifications will be referred to as "approach" here.

It is noteworthy that none of the resulting combinations can be considered non-meaningful a priori. Hence, covering the full multiverse of plausible combinations is key as only a systematic investigation of these combinations will allow us to potentially identify individual specifications, or patterns, that may result in disagreement between different approaches. We highlight that we consider this a case of decision uncertainty (Type $U$ decision according to DelGuidice \& Gangestad, 2021) as we have no empirical evidence with respect to which combination is more valid. To this end, the multiverse-style of investigation presented here is deliberately explorative in nature.

In addition to these $n$ BLC specifications, single trial SCRs were quantified through a prototypical TTP approach for all participants (see Kuhn et al., 2021 and details above), which was included as a reference approach to the multiverse.

Main effects of successful fear acquisition training, i.e., CS+ > CS- responding, will be estimated for all approaches including the TTP approach with Bayesian paired sample t-tests using 100.000 instead of the pre-registered 1.000.000 iterations due to computer resources. A specification curve (Simonsohn et al., 2020), generated through a custom-made R script (available at https://github.com/rsjouwerman/repl-ttpblc) will be used to illustrate the range in effect sizes resulting from the different specifications. The abovedescribed criteria (see 2.3) will be applied to infer whether the $n$ approaches can be considered replications of each other as they all originate from the same set of raw data.

\subsection{Understanding 'negative' SCRs generated by the BLC approach}

Furthermore, we address two issues that potentially arise when using BLC methods: physiologically implausible negative responses (see introduction) and potentially erroneously quantifying a response in the absence of a phasic response. First, we evaluate how frequent negative values per stimulus type in BLC methods are observed, separately for SCR and SCL. The cases in which such negative "responses" are observed will then be compared to the TTP values on those same trials. Frequencies are compared using a chi-square test using the chi-square function in R. Second, to explore whether a potential habituation drift is selected in the PDW rather than an actual stimulus-induced response, the time at which the peak occurs is extracted to infer whether the distribution of the peak-times per stimulus type in the PDWs is possibly left or right skewed. This would be the case when the maximum skin conductance value in the PDW occurs at the value representing the PDW offset (in case of a positive drift) or onset (in case of a negative habituation drift). This is relevant for peak response selection (i.e., SCR) approaches only.

\subsection{Software}

BLC applied to the continuous SC signal for the nine exact specifications from the literature as well as for the full data multiverse, are computed using Matlab version 2018a. Statistical analyses and data 
visualization are conducted in $\mathrm{R}$ version 4.0.4 (2021-02-15). The following packages are central to our analyses and data visualisation: BayesFactor (Morey \& Rouder, 2018) for bayesian paired t-testing, ggalluvial (Brunson, 2020) for flowchart diagram, ggplot2 (Wickham, 2016, S. 2) for plotting, patchwork (Pedersen, 2020) for arranging plots, magick (Ooms, 2021) for making gif. In contrast to the preregistration, the MPTmulitverse package that is optimized for multiple modeling (Bayesian vs NHST) and data pooling choices was not used, instead a specification curve of effect sizes was generated for visualization using the ggplot 2 package that was more feasible for illustrating multiple data choices with only one analysis. 


\section{Results}

\subsection{Systematic literature search}

As described in detail in our related work (Kuhn et al., 2021), the systematic literature search comprised 50 records from which the employed SCR quantification approaches were extracted. TTP scoring $(n=24)$ and baseline correction (BLC) approaches $(n=18)$ were most abundant while model-based approaches ( $n=5$; Ledalab and SCRalyze/PsPM) were less commonly used. Three additional records were excluded as described below. In this previous related work, we reported a direct comparison of SCRs quantified through seven different SCR response quantification approaches (two TTP raters, exemplary BLC approach, Ledalab's CDA, PsPMs single-trialGLM as well as three different DCM-based models in PsPM) as identified by the literature search in the same data as used here as well as an additional dataset not used here (Kuhn et al., 2021).

In contrast to the between-approach comparison reported in our previous work (Kuhn et al., 2021) the present work focuses on heterogeneity in specifications within the BLC category (within-approach heterogeneity) in comparison to one prototypical TTP approach which serves as a reference. All of the 18 studies identified through the systematic literature search, except one using ANSLAB (Rattel et al., 2019) used a custom-made script whereof three studies (Ahmed \& Lovibond, 2019; Lee et al., 2018, 2019) explicitly reported computing SCL rather than SCR. Yet, we excluded one study that investigated SCL without a baseline correction (McGlade et al., 2019), and one study that did not report any detail on SCL quantification (Kurayama et al., 2019). Additionally, one study did not provide any methodological details on SCR quantification as they failed to observe differential SCR in their study and hence results concerning SCR were excluded from their manuscript (Scheveneels et al., 2019) as well as from our literature description.

Remarkably, the frequently used categories of approaches - in particular BLC - were themselves quite inhomogeneous. More precisely, different parameters were applied to define a response such as the OLW for TTP and the PDW or the BLW for BLC approaches. These parameters, however, come in different specifications across studies, which is likely to be partly attributable to different procedural details (e.g., CS and ITI durations). In the present work, we focus on heterogeneity within the BLC approach as identified by the systematic literature search.

\subsubsection{Heterogeneity of parameter specifications within the BLC approach in the reviewed literature}

Four parameters were identified to be used in quantifying skin conductance data in BLC approaches with their specifications differing between the studies included in the systematic literature search (see also Figure 1):

1) The Baseline window (BLW) defines a pre-stimulus time window during which the skin conductance values are averaged to yield a pre-stimulus baseline value. This time window spanned 1, 2, 4, or 10s prior to CS onset until CS onset.

2) The start of the peak detection window (PDW start) which varied between stimulus onset and $3 \mathrm{~s}$ after stimulus onset (i.e., $0,0.5,1,2$, or 3 s post-CS onset).

3) The end of the peak detection window (PDW end) which varied between -1 and 8 s relative to stimulus offset $(-1,0,0.5,1$, or 8 s to CS offset). 
4) The aggregate function (function) applied over all values within the PDW (i.e., extracting the peak response (maximum) in case of SCR or average value (mean) in case of $\mathrm{SCL}$ ).

For the US, four studies explicitly defined quantification parameters. The BLW was either identical to the BLW used to quantity the preceding CS+ response (Gruss \& Keil, 2019; Rattel et al., 2019), or was defined of equal duration as for the BLW of the CS but during a pre-US time period (Geller et al., 2019; Marin et al., 2020), in other words during CS+ presentation.

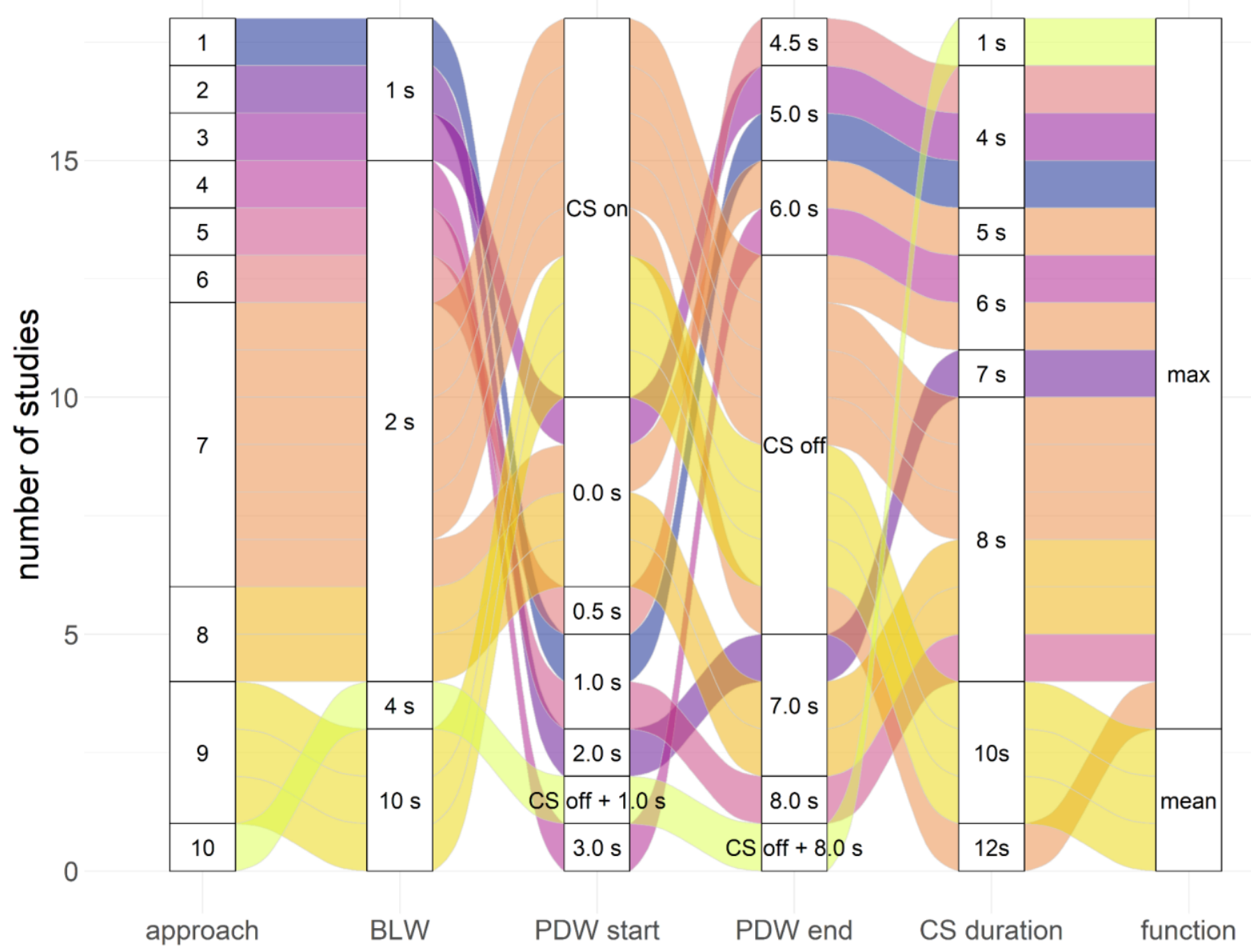

Figure 1. Flowchart of parameters, their specifications and their combinations in the 18 studies using a BLC approach for SCR/SCL response quantification as derived from the systematic literature search. Note that some studies included multiple substudies that used the same criteria. These substudies are not listed separately here and one 'flow' represents details from one publication irrespective of the number of substudies in case identical criteria were used.

Note that the 18 different studies are reduced to ten unique approaches when applying the criteria used in the respective publications to the timing of our experiment. For instance, approach $N^{\circ} 7$ comprises six different publications; all use a BLW of $2 \mathrm{~s}$ and a PDW start at CS onset (i.e. Os). Yet, the PDW end differed between these six studies with one study using a PDW end time at $6 s$, five studies using a PDW end at CS offset. CS duration and hence CS offset, however, varied between 6 and $12 \mathrm{~s}$ in these studies. When translating and applying the criteria in these approaches to our own data with a minimum CS duration --and hence CS offset- of $6 s$, these approaches become identical when applied to our experimental design.

Note that the times (in s) listed for PDW start are with respect to stimulus onset (i.e., CS onset $+X$ s). PDW start times at CS on and at $0 \mathrm{~s}$ thus both correspond to CS onset, i.e., CS onset $+0 \mathrm{~s}$.

Figure 1 illustrates the different parameters as well as their specifications as identified from the 18 records from the systematic literature search that employed a BLC. The ten unique combinations of parameters 
(i.e., approaches) resulting from translation to our experimental timings are color coded and assigned numbers from 1 to 10. Initially, 16 unique approaches out of 18 studies were pre-registered resulting from removal of duplicates $(n=2)$ with the same specifications. Here, six additional approaches were removed because they resulted in duplicated BLC formulas when specifications were applied to our data, i.e., overlap between our CS duration (i.e., 6s) and a PDW-end of $6 \mathrm{~s}$ listed in the literature.

Of note, approach 10 is unique in selecting a PDW starting after CS offset, which is likely due to their short CS presentation time (1s). However, such a strategy should be applied with caution. For the CS+, it is common that the US co-terminates with CS offset, which is also the case in the respective study (Yoshiike et al., 2018). Thus when starting the PDW after CS offset, it is likely that the physiological response to the US will be quantified, rather than the response to the CS+. Therefore such a strategy is likely to overestimate CS discrimination as high US responses will be (miss)attributed to the CS+ which artificially boosts CS+ responses, particularly for $100 \%$ reinforcement paradigms such as in Yoshiike et al. (2018). To avoid this a priori bias in our data analysis, we excluded approach 10 from our further analyses.

For the remaining specifications, we have no compelling reasons to either expect equivalence or nonequivalence between these different methods.

This leaves nine unique combinations of values within the four parameters for our subsequent analyses. As evident from Figure 1, a BLW of 2 s prior to CS onset was most abundant and the PDW most frequently started at CS onset and ended at CS offset. Furthermore, all studies focusing on SCR's used the peak value as the aggregate function, while the three studies focusing on SCL used the average value in the PDW.

\subsection{Main effect of fear acquisition training}

To further our understanding if and to what degree results derived from the different BLC specification combinations and from one prototypical TTP approach converge, we focus on the effect of main interest during fear acquisition training (i.e., CS discrimination). We here provide descriptive and statistical demonstrations on i) the trial-by-trial un-transformed SCR values across the different stimulus types (ie., CS+, CS-, US, Figure $\mathbf{2}$ A, B and C), ii) average values for each stimulus type (Figure 2D), iii) CS discrimination (i.e., CS+ - CS-, Figure 2E) as well as iv) the respective effect sizes derived from bayesian paired T-tests (Figure $\mathbf{2 F}$ ). This is shown across the nine unique BLC approaches and the prototypical TTP approach.

Unsurprisingly, the different approaches result in different numerical values for trial-wise (Figure 2A,B and C), averages per CS type (Figure 2D) and CS discrimination (Figure 2E) during acquisition training. Yet, the descriptive trajectory over the 14 fear acquisition trials seems largely convergent across approaches. Bayesian paired-sample-t-tests for average CS discrimination (i.e., CS+ vs. CS-) during fear acquisition training (excluding first trial per CS) reveal evidence for an effect of stimulus type, with CS+ responding exceeding CS- responding, and with large effect sizes in all approaches (see Figure $\mathbf{2 F}$, all $\mathrm{BF}_{\ln \mathrm{S}}$ $>$ 37). Thus, we can conclude that there is a signal (LeBel et al., 2020) irrespective of the unique combination of BLC parameter specification. Of note, effect sizes are consistent across all approaches for all pairwise comparisons, indicated by the point estimate of each approach falling within the $95 \% \mathrm{Crl}$ of any of the other approaches (LeBel et al., 2020). 


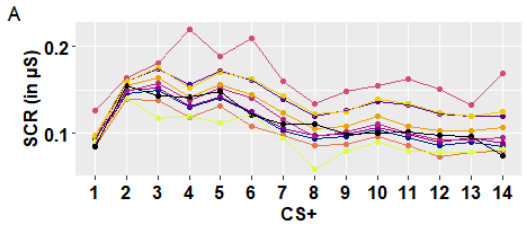

B
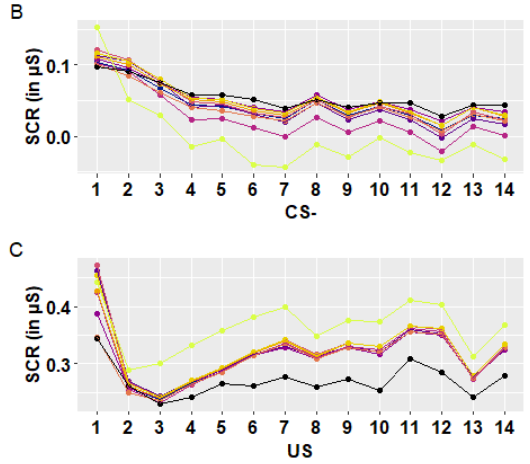

$\mathrm{D}$

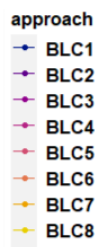

BLC9

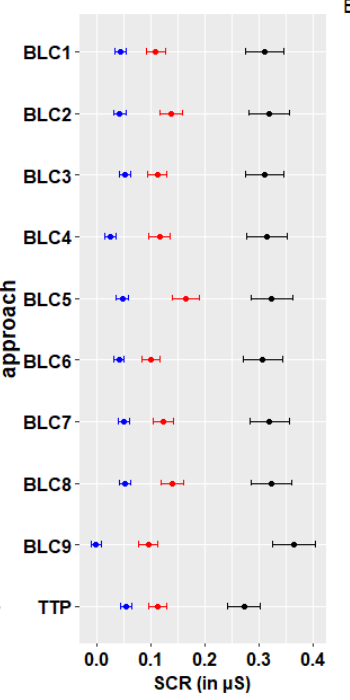

E

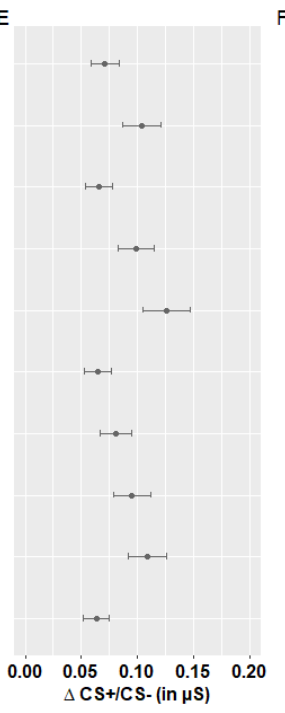

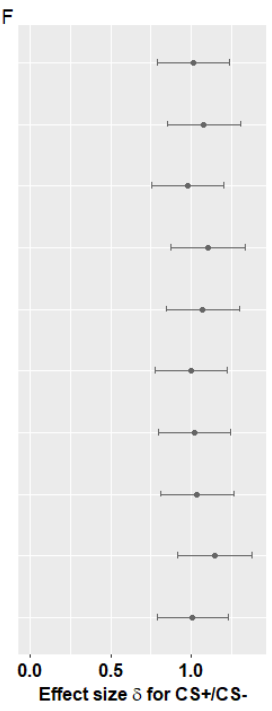

Figure 2. Skin conductance data (un-transformed values) derived from all nine BLC approaches and the prototypical TTP scoring approach, illustrated for single trial data averaged over participants for 14 fear acquisition training trials separately for CS+ (A), CS- (B) and US (C). Averaged skin conductance per stimulus type (CS+ in red, CS- in blue and US in black) for each of the ten BLC and the one TTP approach (D) as well as the difference between averaged CS+ and CS- (i.e., CS discrimination) across acquisition training (E). Effect sizes of corresponding bayesian paired-sample-t-tests for all approaches (F). Error bars represent $95 \%$ credible intervals in $D$ and $E$, credible intervals in $F$.

In sum, average CS discrimination with consistent effects sizes is observed across the nine unique parameter specifications derived from the literature. Yet, this focus on average responding across participants may obscure potential differences between approaches on an individual level. Next, we evaluate whether the different approaches affect individuals (individual ranking), and trials differently.

\subsection{Trial-by-trial rank stability between approaches}

The convergence or divergence of the nine BLC approaches and one prototypical TTP approach are evaluated in terms of agreement across and between approaches during fear acquisition training (14CS+, 14CS-, 14US). Trial-wise Krippendorff's $\alpha$ is estimated as an index for the individuals' rank stability across BLC approaches as well as pairwise between all possible pairs of nine BLC and one TTP approaches.

Comparing all nine BLC approaches in a single analysis (Figure $3 \mathrm{~A}$ ) results in substantial to near perfect overall agreement with trial-wise Krippendorff's $\alpha$ estimates varying between 0.69 and 0.98 . Highest values are observed for USs, and lowest values for CS-s (estimate mean [min - max] for CS+: .89 [.85 - .97], CS-: .80 [.69 - .88], and US: .96 [.93 - .98]). This indicates that on average an individual keeps its rank more accurately for US and CS+ responses across approaches (i.e., perfect agreement), whereas rank stability, and thus agreement, is only moderate between approaches for the CS-s. 
B
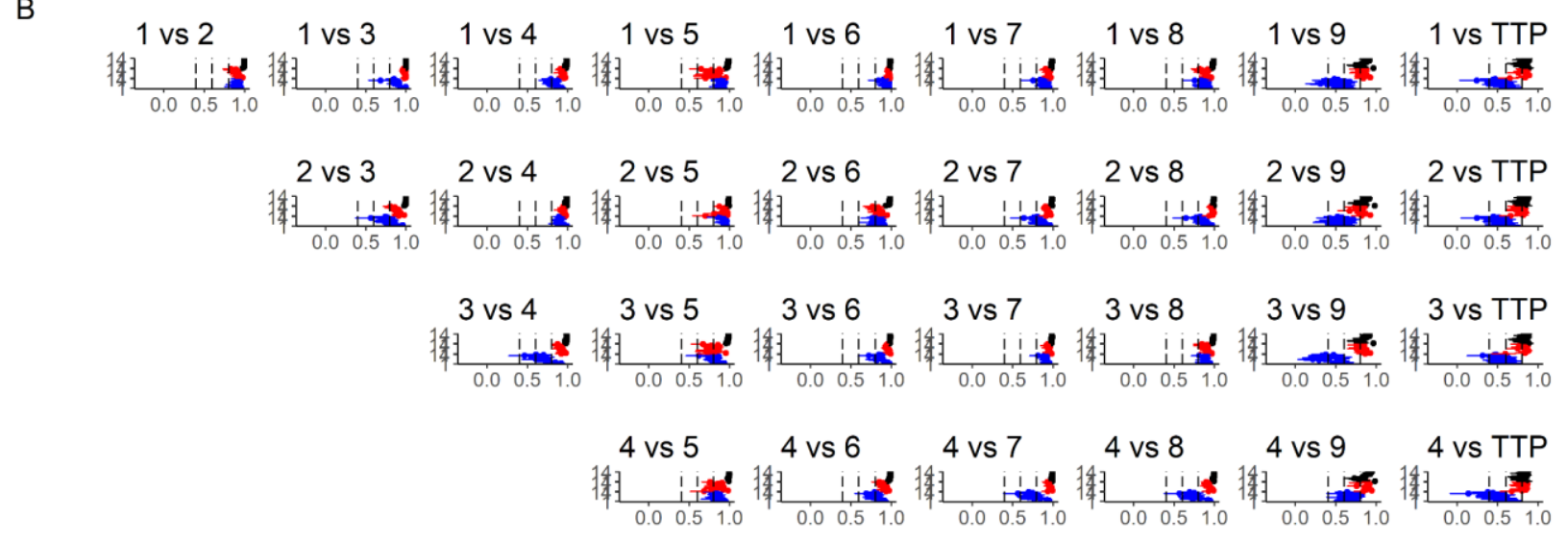

A

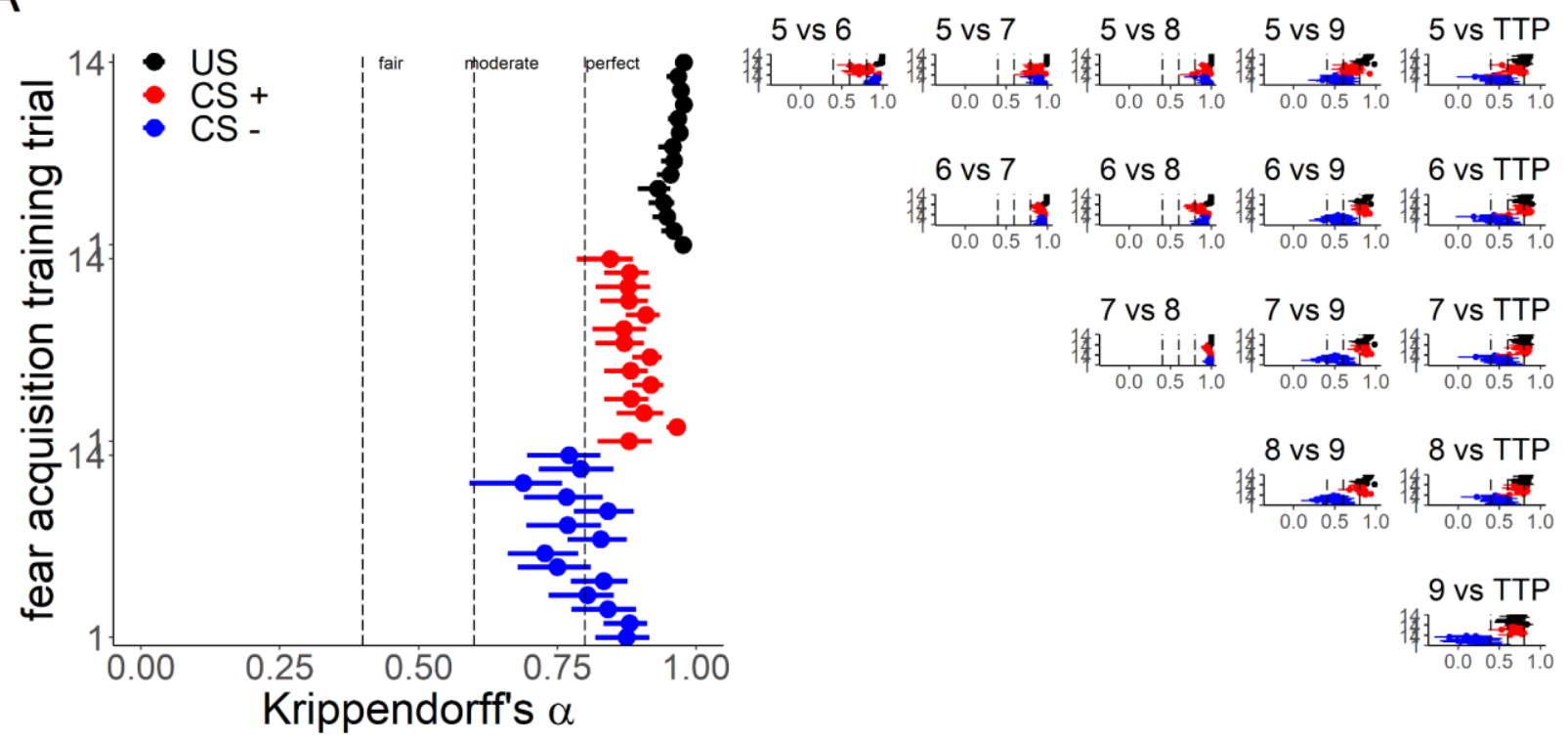

Figure 3. Agreement indicated by Krippendorff's $\alpha$ and 95\% bootstrapped confidence intervals for the 14 trials per CS+ (red), CS(blue) and US (black) during fear acquisition training across all nine BLC approaches (A) as well as pairwise comparisons between all BLC approaches and between all BLC approaches and the TTP approach (B).

Pairwise-comparisons between all approaches (i.e., BLC and TTP) illustrated in Figure 3B highlight that agreement was almost perfect between some pairs of approaches (e.g. BLC1 and BLC2, BLC7 and BLC8) while it was modest between other pairs of approaches particularly for CS- trials (e.g., BLC3 and BLC4, BLC6 and BLC9). Remarkable high agreement was observed for BLC7 vs BLC8, approaches that differ only in the post-CS end time of the peak detection window (i.e., CS offset at $6 \mathrm{~s}$, or $1 \mathrm{~s}$ after CS offset at 7s), suggesting that peaks mostly occur prior to $6 \mathrm{~s}$ and are hence identified comparably irrespective of length of the PDW with respect to its end time point.

Notably, pairwise comparisons with BLC9, which explicitly focuses on SCL instead of SCR, are marked by relatively wide confidence intervals which results in less precise reliability estimates. Excluding BLC9 - which focuses on SCL - from estimation of Krippendorff's $\alpha$ across approaches (Figure 3A) hence unsurprisingly increased overall agreement. Yet, the pattern of a lower rank stability, i.e., lower agreement for CS-s observed with all nine BLC approaches remains when BCL9 is excluded (see Supplementary Figure 1). 
Comparisons between BLC approaches and the one TTP approach, see last column in Figure 3B, are also marked by relatively wide, in other words imprecise, confidence intervals irrespective of stimulus type. In addition, for CS- trials the agreement is low between any of the BLC and TTP comparisons, while agreement for the CS+ and US is in a moderate to substantial range (see Supplementary Table 1 for details). Specifically for SCL (BLC9) and TTP, estimates center around and even go below zero for CS- trials indicating poor agreement or even disagreement between both approaches (mean estimates [min - max] for CS-: .22 [-.11 - .49], CS+: .71 [.53-.79], and US: .71 [.60 - .84]).

\subsection{Covering the full data multiverse of parameter specifications}

The specifications identified from the systematic literature search (see Figure 1) while excluding approach 10 (for reasons see above) give rise to 150 possible unique combinations of values derived from the four identified parameters (i.e., BLW, PDW-start, PDW-end, aggregate function).

\subsubsection{Main effect of fear acquisition training in the data multiverse}

Bayesian paired-sample-t-tests reveal evidence for a main effect of stimulus type in all 150 BLC approaches and in the TTP approach, all $\mathrm{BF}_{\ln S}>$ 34. CS+ responding exceeded CS- responding in all 151 cases (i.e., evidence for CS discrimination). A specification curve of the effect sizes for CS discrimination across the 151 approaches is displayed in Figure 4 and an animated digital version highlighting a color-coded arrangement of the four parameters included in the specification curve (BLW, PDW-start, PDW-end, aggregate function) is available as Supplementary material figure 2. Effect sizes are ordered by their magnitude in the specification curve and it is noteworthy that all 151 effect sizes are large with a range between 0.97 and 1.23. Interestingly, the effect size of the TTP approach is on the lower end of the curve, and almost all (i.e., 141 of 150) BLC approaches have larger effect sizes.

Inspecting the specification curve (see also online available color coded Supplementary figure 2) suggests tendentially larger effect sizes for BLC methods where the mean rather than the maximum value is selected in the PDW - that is when SCL rather than SCR is the target. Furthermore, combinations including longer BLWs also seem to result in slightly larger effect sizes. Interestingly, effect size estimates of BLWs of 10 s tend to result in the largest effect sizes, possibly explained by the average of the BLW being less affected by extreme signal fluctuations, which consequently puts more weight on the value that is selected in the PDW. Similarly longer PDW windows seem to contribute to slightly larger effect sizes as well.

Yet, despite these descriptive patterns, almost all effects can be considered as replications of the effects in approaches with different specifications. For example, the largest effect size estimate (approach with a BLW of 10s) still falls within the $95 \%$ boundaries (i.e., Crl) of nearly all (except the seven smallest) effect sizes of approaches with shorter BLWs. 


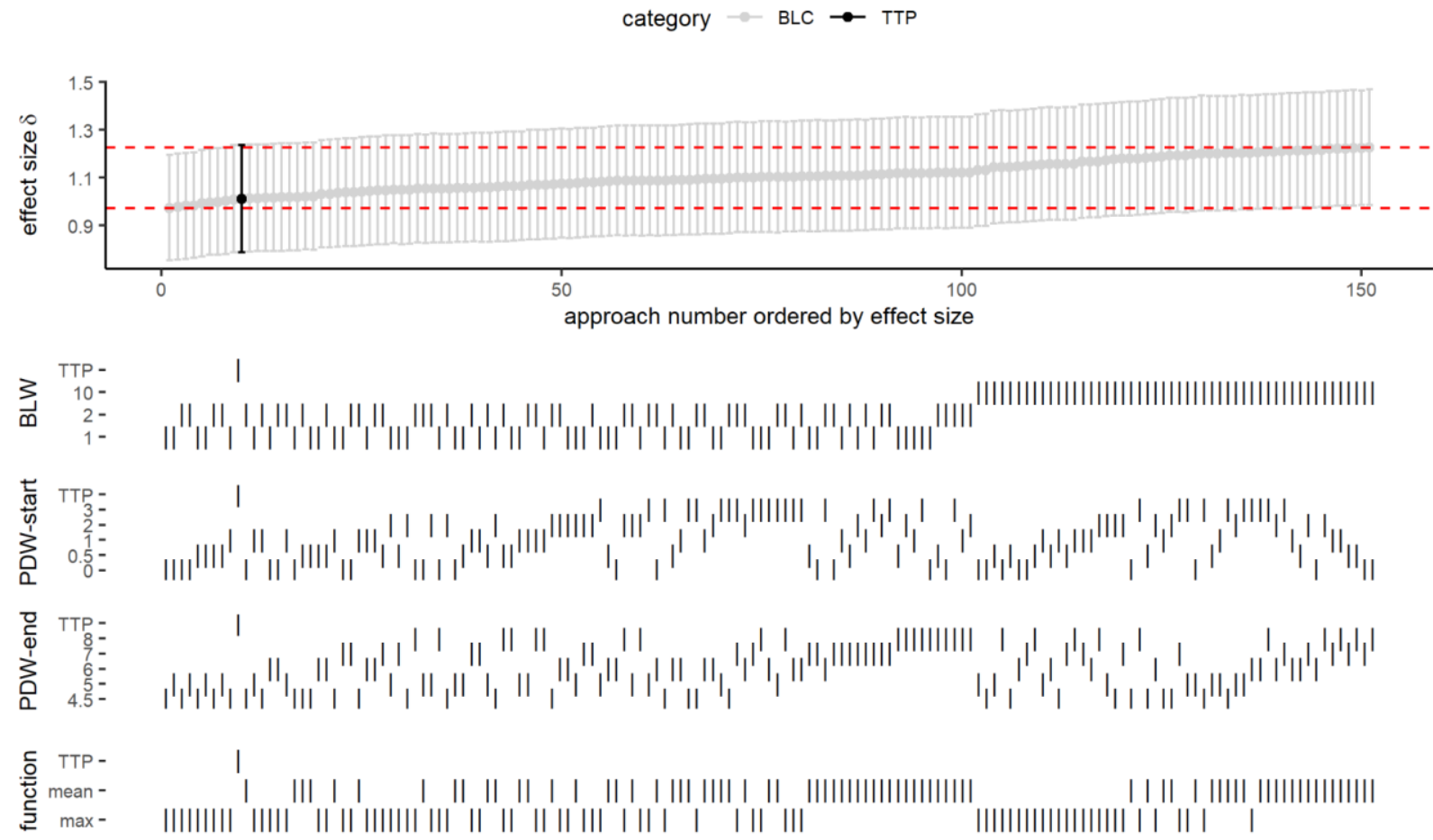

Figure 4. Specification curve of effect sizes $\delta$ of bayesian paired-sample-t-tests on the main effect of CS discrimination for 150 BLC approaches (grey) and one TTP approach (black) as reference in the top panel. Effect sizes are ordered by increasing magnitude. Red dashed lines indicate the range of maximum and minimum median effect sizes. The lines below indicate the specification of each of the four parameters behind the effect size estimates. BLW = baseline window; PDW-start = peak detection window start time: $P D W$-end = peak detection end time; function = aggregate function used in PDW.

\subsubsection{How do 'negative' values in BLC approaches correspond to values in the TTP approach?}

One crucial difference between SCR quantification through TTP and BLC approaches is that TTP scoring can only yield positive values while BLC yields negative values. This occurs for instance when the value during the BLW exceeds the peak value detected during the PDW, when the response is small in respect to a strong habituation drift or when no response occurs and only a habituation drift is present. Yet, it remains unexplored how frequently negative values occur when using BLC approaches, whether they are stimulus dependent and how to interpret these negative values with respect to the TTP approach in which negative SCR values are (physiologically) implausible. Here, we address these questions empirically.

Values derived from the averaged BLWs in the 150 BLC approaches exceeded the extracted peak value in the PDW in $23.2 \%$ and $39.7 \%$ of all trials for the maximum (i.e., SCR; $B L C_{\max }, n_{\text {approach }}=75$ ) and mean (i.e., $\left.\mathrm{SCL}, \mathrm{BLC} \mathrm{mean}_{\text {n, }} \mathrm{n}_{\text {approach }}=75\right)$ functions respectively. Irrespective of maximum $(\mathrm{SCR})$ or mean $(\mathrm{SCL})$ function in the PDW, most of these negative values are observed in response to the CS- (in blue, BLC $\mathrm{max}_{\mathrm{m}} 68.7 \%$ and $B L C_{\text {mean }}: 60.1 \%$ ), with approximately half as much negative responses to the $\mathrm{CS}+$ (in red, $\mathrm{BLC}_{\text {max }}: 30.9 \%$ and $B L C_{\text {mean }}: 35.7 \%$ ) and very few in response to the US (in black, $B L C_{\max }: 0.4 \%$ and $B L C_{\text {mean }}: 4.2 \%$; see Figure $5 \mathrm{~A}$ and $B)$.

A chi square test supports that the proportion of CS-s is largest, followed by CS+s, and smallest for USs in both SCR $\left(\mathrm{chi}^{\wedge} 2(2)=78651.41, \mathrm{p}<.001\right)$ and SCL approaches $\left(\mathrm{chi}^{\wedge} 2(2)=114989.44, \mathrm{p}<.001\right)$. US responses rarely result in negative values with the applied BLC operationalizations, but their frequency is higher when 
$\mathrm{SCL}$ is the target (Figure 5A) as compared to when SCR is the target (Figure 5B) $\left(\right.$ chi $^{\wedge} 2(1)=5416.12, p<$ .001). In addition, frequencies of negative values also differed between SCR and SCL approaches for CS+ $\left(\right.$ chi^$\left.^{\wedge} 2(1)=12613.81, p<.001\right)$ and CS- $\left(\right.$ chi^$\left.^{\wedge} 2(1)=14541.82, p<.001\right)$. Of note, $98 \%$ of all individuals showed at least one negative SCR for $B L C_{\max }$ and for $B L C_{\text {mean }}$ all (i.e., 100\%) individuals showed at least one negative SCR. Negative SCRs thus do not seem to be a peculiarity observed for individual subjects only.

A

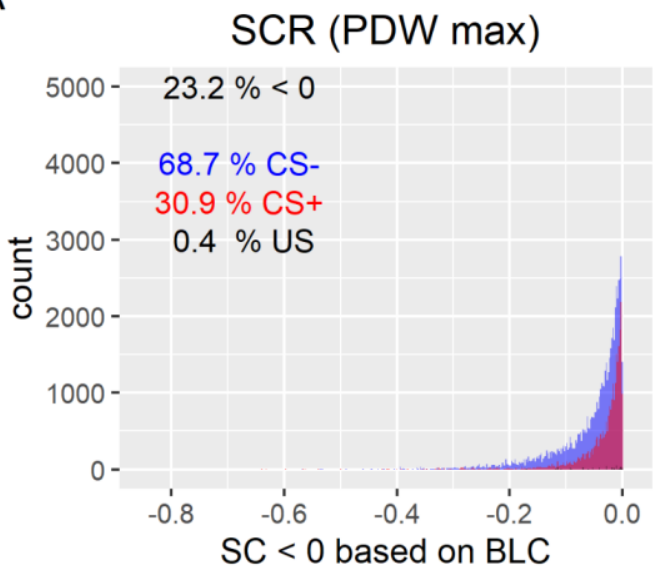

C

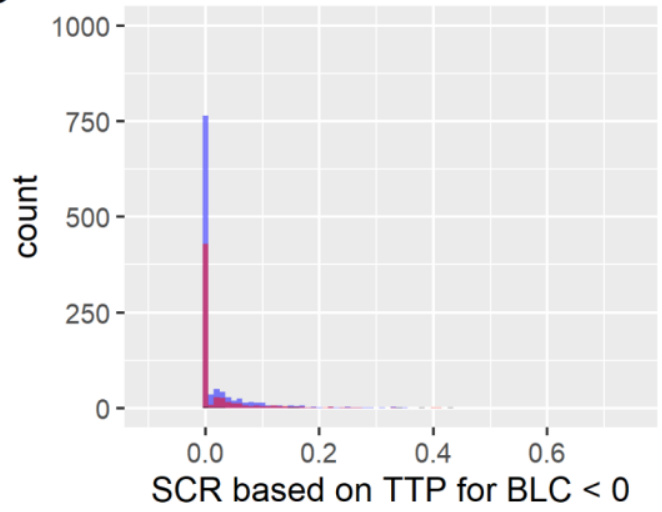

B
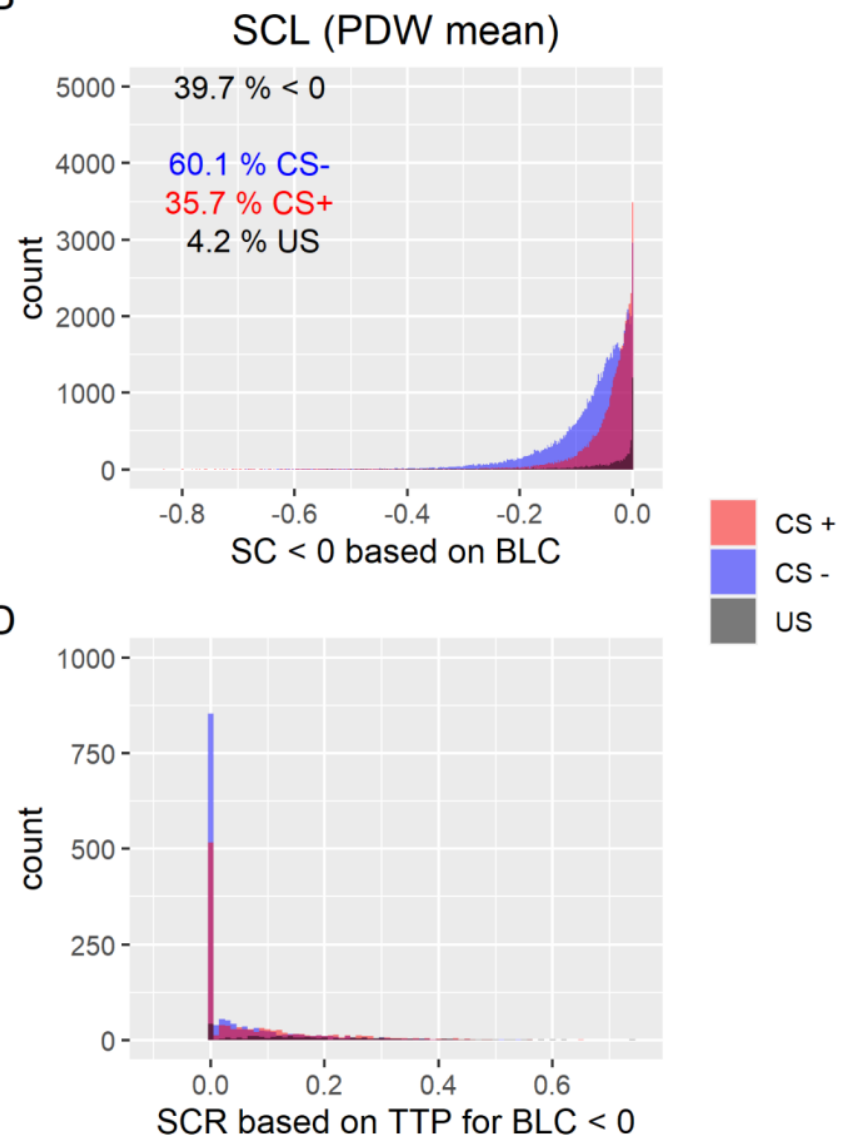

Figure 5. Overlaid histograms of skin conductance values below zero (i.e., negative SCR) as identified by any of the 150 BLC methods included in the multiverse illustrated for the three stimulus types CS+ (red), CS- (blue) and US (black). Results are depicted separately for (A) data derived from BLC approaches using the maximum peak skin conductance value during the PDW (i.e., SCR) and (B) for those methods using the average skin conductance value (i.e., SCL) during the PDW. Furthermore, we illustrate (C) a histogram of SCR values quantified through the TTP approach that correspond to the specific trials where a negative SCR was quantified with $B L C s$ using the maximum peak skin conductance value (i.e., SCR) during the PDW (D) or the average skin conductance value during the PDW (i.e., $S C L)$.

To further our understanding of what these negative values reflect, we compared the negative skin conductance values as quantified by the multiverse of BLC approaches with the SCRs in the corresponding trials as quantified through the TTP approach. Most of these negative values derived from the approaches using the aggregate function to find the maximum value (i.e., SCR) correspond to zero-responses in the TTP method (69.1\%), mostly CS- trials (63.9\% CS-; 35.8\% CS+; <1\% US), chi^2(2) = 731.01, p <.001. Negative values derived from the approaches using the mean aggregate function (i.e., $\mathrm{SCL}$ ) also often (48.7\%) correspond to zero responses in the TTP method, again most of them in response to CS-s (60.4\% CS-; $36.6 \%$ $\mathrm{CS}+; 3.0 \% \mathrm{US}), \operatorname{chi}^{\wedge} 2(2)=706.68, \mathrm{p}<.001$. Interestingly, these negative values for the mean aggregate function (i.e., SCL) correspond in more than half of the cases to actual positive SCR values in TTP scoring - 
not to zero-responses or missings - and thus potentially contribute to divergent results between $\mathrm{SCL}$ approaches using a BLC and BLC or TTP approaches for SCR. Note also that the percentage of trials explicitly scored as 'missing' by using the TTP approach for these values is $<1 \%$ (for both mean or maximum aggregate function).

\subsubsection{Revisiting drift selection instead of response selection in BLC approaches}

The different BLC approaches focusing on SCR (i.e, using the maximum aggregate function) identified through the literature search all used fully automated script-based quantification of SCRs. None used an iterative algorithm that in the original article (as in (Pineles et al., 2009), see also description in the introduction) ensured that the identified peak value in fact represents a peak rather than just the first value in the PDW that may only show a habituation drift (or the last value in case of an increasing drift). Such a first (or last) value would not be indicative of a stimulus-induced SCR but would in fact represent a non-response (i.e., only a habituation drift in skin conductance). To investigate if and how often the different BLC approaches select a peak that in fact represents the first or last value in the PDW and thus may represent merely a habituation drift rather than a stimulus-induced SCR, we extracted the time-point at which the selected peak occurred for all approaches from the multiverse that used the maximum aggregate function (i.e., SCR).

The distribution of time-points of selected peaks for the CS+ (red), CS- (blue) and US (black) are illustrated for all 25 different PDWs as defined by combinations of the PDW starting times (i.e., Stimulusonset, Stimulus onset $+.5 s$, Stimulus onset $+1 s$, Stimulus onset $+2 s$, Stimulus onset $+3 s$ ) and PDW end times (i.e., Stimulus offset $-.5 \mathrm{~s}$, Stimulus offset $-1 \mathrm{~s}$, Stimulus offset, Stimulus offset $+1 \mathrm{~s}$, Stimulus offset $+2 \mathrm{~s})$ in Figure 6 . Note that stimulus offset for the US equals stimulus onset due to its short duration and would render implausible time windows with PDWs "ending" before they have "started". In order to compare time windows for the US also to CS time windows an arbitrary "stimulus offset" of $6 \mathrm{~s}$ was used that corresponds to the mean CS duration and thus is the same as for CSs. The long ITI duration of the current experiment (10-16s) exceeds the CS duration in time and makes this feasible.

For the US, it is striking that time points of peak occurrence are normally distributed with the exception of PDWs starting $3 \mathrm{~s}$ after US onset (last row in Figure 6). This likely indicates that the peak response might be missed as US responses often start very early and peak before 3s post US onset (Sjouwerman \& Lonsdorf, 2019).

For the CS+ and the CS-, however, the time points of peak occurrence are not normally distributed for any of the combinations of PDW start and end times. For the CS- time points of peak occurrence are primarily right skewed while time points of peak occurrence for the CS+ are skewed to both ends across all 25 PDWs -though with a stronger tendency to be left skewed. For the CS-, the right skewed distribution most likely illustrates that the first value in the PDW is often erroneously selected to represent the SCR peak, which may indicate that these cases indeed may represent trials in which only a habituation drift but no stimulusinduced SCR is observed. For the CS+ the same explanation likely applies to the part of the distribution that is right skewed. A substantial part of the distribution of peak time points for the CS+ are also leftskewed. It can be speculated that these values may represent anticipatory (i.e., second interval) responses or an upwards drift in SCL. 


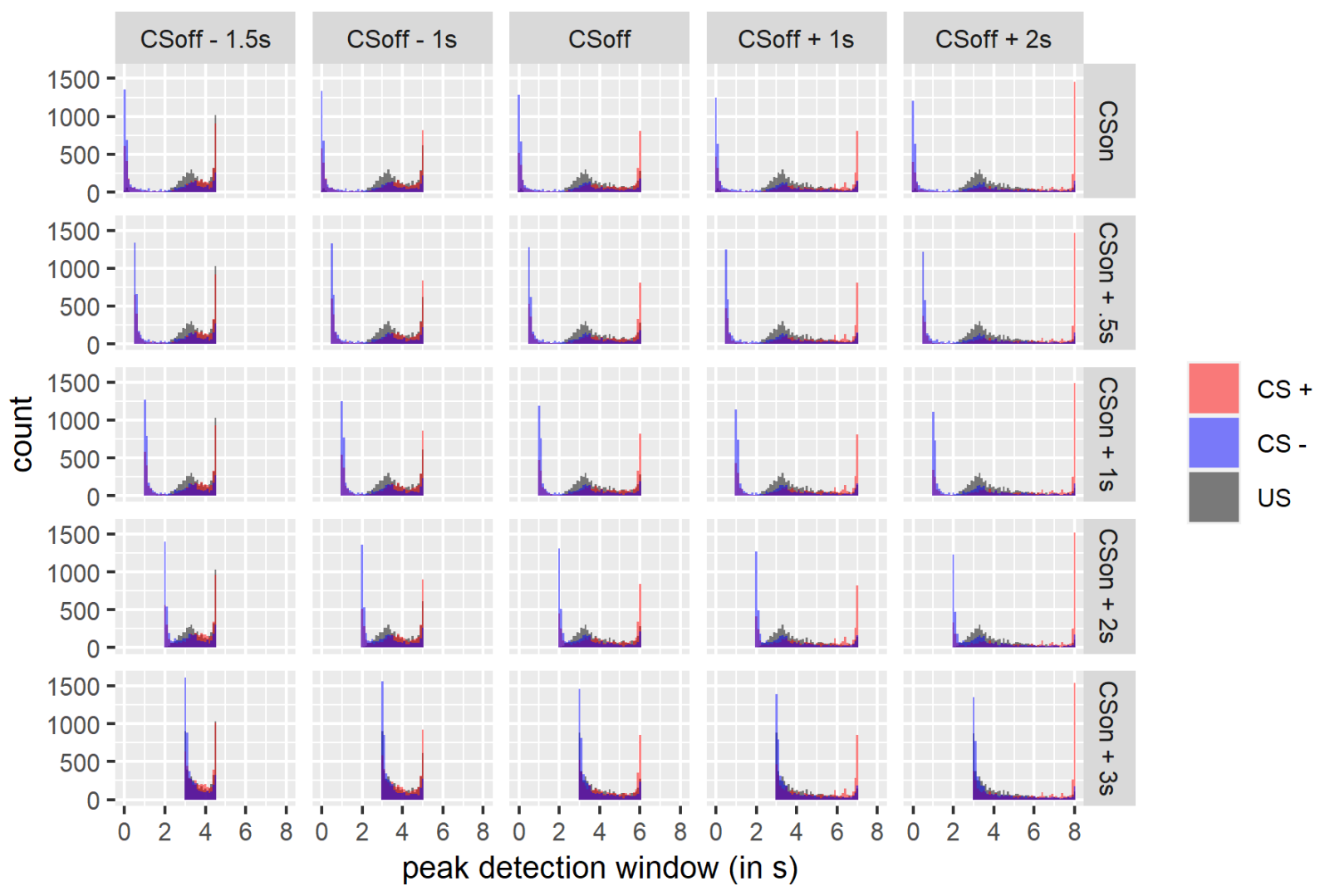

Figure 6. Histograms of the onsets of the selected maximum peak skin conductance value within the 25 different PDWs derived from combinations of PDW start and end times illustrated for the three stimulus types (CS+ in red, CS- in blue, US in black). The 25

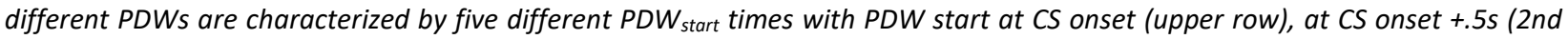
row), at CS onset $+1 \mathrm{~s}$ (3rd row), at CS onset $+2 s$ (4th row) and CS onset $+3 s$ (bottom row) as well as five different PDW end $_{\text {times, }}$ with PDW endsat CS duration -1.5s (1st column), at CS duration -1s (2nd column), at CS offset (3rd column), at CS offset $+1 \mathrm{~s}$ (4th column), and at CS offset $+2 s$ (5th column). 


\section{Discussion}

Here we present a multiverse analysis evaluating the replicability of results derived from different nonmodel-based skin conductance response quantification approaches by using fear conditioning as a case example. In contrast to our recent complementary work (Kuhn et al., 2021) we do not focus on betweenapproach comparisons but focus on within-approach heterogeneity for the BLC approach. First, we investigate nine unique baseline-correction (BLC) approaches as identified from the literature and second, we provide a full multiverse approach based on all meaningful combinations $(n=150)$ of the identified specifications. We use a prototypical TTP approach for skin conductance quantification as a reference and apply all resulting 151 quantification approaches to a single set of raw data $(\mathrm{N}=118)$. In sum we observe generally strong agreement across results derived from different BLC approaches and with results derived from the TTP approach, which is generally reassuring. Crucially, however, we also highlight differences in terms of (stimulus-specific) disagreement and substantial problems when using BLC approaches that together call at least for caution and may even speak against their employment.

\subsection{Do different operationalizations of BLC-based skin conductance response quantification converge?}

The systematic literature search revealed that BLC-based and TTP-based skin conductance quantification approaches were most commonly used. Furthermore, substantial cross-study heterogeneity in parameter specification (i.e., BLW, PDW-start, PDW-end, function) in BLC-based quantification approaches was evident - we identified a set of ten unique combinations of parameter specifications. We applied nine of these to a single set of raw data to investigate whether results derived from these different approaches converge and can be considered as (direct) replications.

Significant CS+/CS- discrimination during fear acquisition training, our effect of main interest, was observed for all nine BLC approaches as well as for the reference TTP approach. Of note, applying a replicability framework suggests that effect sizes derived from these in total ten different skin conductance quantification approaches can be considered (direct) replications of each other as all point estimates fall within the other approaches' credible intervals (LeBel et al., 2018). This might be taken to suggest that it would indeed be justified to treat these different approaches interchangeably in the literature and interpret them as 'direct replications'. Yet, whether this interpretation generalizes to more subtle effects (e.g individual differences) is an empirical question that future work should investigate in depth.

Furthermore, an exclusive focus on effect size estimation is insufficient, as our reliability (agreement) analyses showcase: More nuanced analyses focusing on trial-wise agreement between the nine BLC-based quantification approaches revealed substantial to near perfect agreement - yet in a stimulus-type dependent way. More precisely, agreement was near perfect for US trials and CS+ trials but somewhat lower for CS- trials (i.e., substantial). This suggests that the nine approaches have little discrepancy in quantifying strong skin conductance responses (i.e., to the US and CS+) while the quantification of the smaller CS- responses differs somewhat more between approaches.

\subsubsection{Pairwise agreement between different BLC approaches specifications can reveal systematic biases.}

Investigation of the pairwise agreement between all pairs of approaches provided a detailed picture and revealed a set of approaches that show near perfect agreement irrespective of CS-type - for instance approach 2 (PDW = 2-7s; BLW = 1s) and 4 (PDW = 3-6s; BLW = 2s) as well as approach 6 (PDW = 0.5-4.5s; $\mathrm{BLW}=2)$ and $7(\mathrm{PDW}=0 \mathrm{~s}-6 \mathrm{~s} ; \mathrm{BLW}=2 \mathrm{~s})$ or 7 and $8(\mathrm{PDW}=0-7 \mathrm{~s} ; \mathrm{BLW}=2 \mathrm{~s})$. The latter two approaches 
for example (i.e., 7 and 8) differ only in PDW length, more precisely the end time of the PDW being respectively at 6 or $7 \mathrm{~s}$ which is unlikely to matter much as the peak is expected to occur earlier than $6 \mathrm{~s}$ or $7 s$ anyways.

Other sets of approaches show low agreement specifically for the CS+ trials - such as approach 5 (PDW = $1-8 \mathrm{~s} ; \mathrm{BLW}=2 \mathrm{~s}$ ) - characterized by the longest PDW - as compared to any other approach. This is unsurprising given that the PDW-end value for approach 5 (i.e., 8s) exceeds our minimal CS duration (i.e.,

6 s minimum) and therefore may capture skin conductance increases attributable to the US presented at CS+ offset at least for our shorter CS durations of $6 \mathrm{~s}$ but less likely for our longer CS durations of $7 \mathrm{~s}$ and $8 \mathrm{~s}$. Note that CS duration in our experiment was jittered between $6 \mathrm{~s}$ and $8 \mathrm{~s}$.

Yet, other sets of approaches show little agreement specifically for CS- trials such as approach 3 compared to approaches 1, 2, and 4, or approach 4 compared to approach 7 and 8, to name just a few examples. Crucially, approach 4,7 and 8 share an identical BLW length (i.e., 2s) and identical PDW endings at CS offset (i.e., 6s) but approach 4 differs from 7 and 8 by a late start of the PDW at 3s after stimulus onset. We speculate that, in approach 7 and 8 a (presumably) stimulus unrelated peak is selected resulting from selecting the first value after CS onset, as can be inferred from the distribution of timepoints of peaks (Figure 6). This is likely reflective of a (strong) negative habituation drift. The (true) peak of the CSresponses, however, is reached on average after the first 3s after CS- presentation (see figure 6, median of CS- peak times $=3.5 \mathrm{~s}$ ). This peak identified by approach 4 must thus necessarily be smaller than the peak in the earlier time window - otherwise approach 7 and 8 would have identified the same peak as approach 4 because all share an identical PDW end. Consequently, we speculate further that the (true) CSpeak is missed in approach 7 and 8 because it is small and does not exceed the maximum skin conductance value at stimulus onset - which however is unlikely to reflect a stimulus-induced response but rather the highest value of a habituation drift. As the first 3 seconds of the stimulus presentation period are not included in the PDW of approach 4, the bias in favor of selecting the largest skin conductance value at the first time-point in the PDW is eliminated and the (presumably) true peak of the CS- response is selected in this later time window.

Notably, pair-wise CS trial agreement was particularly low between approach 9 which targets SCL by using the average value during the PDW and any of the other approaches which target SCR by using the peak value during the PDW. While agreement for CS+ and US was in the range of substantial perfect between approach 9 and any other approach ( mean and range estimates CS+: 0.83 [0.61 - 0.94; US: 0.87 [0.73 0.99]), CS- based values were only fair to substantial between approach 9 and the other approaches (range CS-: 0.55 [0.21-0.79]). The substantial agreement for CS+ and US between SCR and SCL approaches is surprising as it suggests that they measure similar processes despite aiming to measure different processes (tonic vs. phasic).

In sum, we clearly show that comparable or replicated effect sizes (as observed here) cannot be taken to infer that any two approaches capture the same underlying process and that results can be used and interpreted interchangeably. It needs to be considered carefully that larger effect sizes are not necessarily always "better" as this may be the result of an 'artifact' and may not be reflective of the true signal.

\subsection{Do BLC-based and TTP-based skin conductance response quantification converge?}

Similarly, pair-wise agreement between responses quantified through the prototypical TTP approach and the nine BLCS approaches revealed generally moderate to perfect agreement for US and the CS+ trials while agreement for the CS- trials was only substantial to poor and in many pairwise comparisons even in 
disagreement. Of note, disagreement with TTP for the CS- was most pronounced for the approach that targeted SCL rather than SCR by using the mean response during the PDW as well as a long BLW of 10 s (approach 9). This supports that SCL as quantified through BLC measures something different than TTP particularly for the CS- trials. Of note, disagreement for CS- quantification was also observed when comparing the eight BLC-based SCR quantification approaches (approach 1-8) to BLC-based SCL quantification (i.e., approach 9). Given that SCL and SCR do not intend to capture the same processes (i.e., tonic vs. phasic), this may not be utterly surprising. Yet, as discussed above, it is surprising and somewhat alarming that the disagreement between the TTP approach and the BLC-based approaches is stimulusspecific (i.e., most pronounced for the CS-). Please note that our previous related work (Kuhn et al., 2021) already reported results for the comparison of TTP with one prototypical BLC approach which we here extend with a focus on within-BLC approach heterogeneity.

\subsubsection{Understanding CS- specific disagreement between TTP and BLC based response quantification}

A potential reason for this CS- specific disagreement may be negative response values which occur in BLCbased quantification but are physiologically implausible when using TTP based SCR quantification as the amplitude cannot be lower than a non-response which is indicated by a zero. BLC-based response quantification, however, can and does yield negative values. Yet, it remains poorly understood how these negative values are to be interpreted.

Negative responses occur when the values during the peak detection window (PDW) are lower than during the baseline window (BLW). Theoretically, this most likely reflects a small phasic response or a nonresponse (i.e., zero) while a strong habituation (i.e., downwards) drift is present. If a small phasic response is present as well as a strong habituation drift, TTP quantification would result in a small non-zero amplitude, while BLC-based quantification would yield a negative value. Similarly, when no phasic response is present, but a strong habituation trend, TTP quantification would yield a zero response while BLC-based quantification would again result in a negative value with the degree of negativity reflective of the strength of the habituation in this trial. This blending of actual response value and habituation strength particularly for small and zero responses may be the reason for the CS- specific disagreement and larger imprecision between TTP and BLC-based approaches.

Indeed, our results show that for SCR, such negative values occur mostly for the CS- (69\%), less often for the CS+ (31\%) and only very rarely for US trials $(0.4 \%)$ and that the major share of negative values corresponds to zero amplitude reactions in TTP - yet, also approximately $30 \%$ correspond to positive TTP values.

Some authors (Mertens et al., 2019; Vogel et al., 2015) routinely replace negative values as derived from a BLC approach with zero but our results highlight that this also sets $30 \%$ of non-zero responses to zero which may again lead to biases. Furthermore, as we have illustrated that BLC based approaches intermix phasic responses with strength of the habituation drift, such a replacement would impact more on CSresponses than on CS+ and US responses which may also be problematic as it impacts on the distribution of the data.

When CS- quantification leads to different results when using different approaches, this consequently impacts on CS+/CS- discrimination also when agreement for CS+ quantification is near perfect. If, CS+ values as derived from TTP and BLC quantification are identical but BLC based quantification results in lower (i.e., negative) values than TTP quantification, this leads to artificially enhanced CS discrimination. Furthermore, the strength of this enhancement effect is critically dependent on the 
strength of habituation with stronger habituation theoretically leading to stronger discrimination as specifically CS- values are lower. Indeed, CS discrimination was numerically larger for all BLC based approaches as compared do the TTP approach - yet, effect sizes for CS discrimination were comparable even though the effect size for TTP quantification was numerically smaller.

Our results highlight that an exclusive focus on higher effect sizes is insufficient and may be misleading as, observing "stronger" effects in the expected direction may not be taken to infer that the effect is 'more true' or reflects a better choice for response quantification.

\subsection{Exploring the full multiverse of BLC parameter specification}

To allow for a more systematic investigation of the impact of different parameter specifications, a full data multiverse analysis included all specifications derived from the literature combined into 150 unique theoretically equally reasonable "approaches". The specification curve illustrated descriptive patterns of the impact of the four parameters used in BLC quantification on the effect size magnitudes. Still, main effects of CS discrimination can be considered as replications of the effect size of the other approaches used.

More precisely, a signal (i.e., significant CS discrimination) was detected with comparable precision and consistency across all approaches (150 BLC +1 TTP) even though longer BLWs, and later starting and later ending PDWs seemed to result in larger effect sizes on a descriptive level. Similarly, on a descriptive level, larger effect sizes were observed for SCL approaches rather than SCR approaches of BLC quantification. Yet, we would like to highlight that the full multiverse of SCR response quantification is substantially larger than what is covered here (e.g., transformations, cut-off criteria, exclusion criteria).

In sum, the convergence of effect sizes across the multiverse of specifications is generally reassuring as it shows that results - on average - are robust across specific operationalizations of non-model-based SCR response quantification even though we would like to point out that there are substantial and concerning problems with the BLC approach to SCR quantification, that we outline in detail in the following.

\subsubsection{Additional problems with BLC based response quantification: implausible peaks}

The original adaptive BLC algorithm used by Pineles (Pineles et al., 2009) used an iterative PDW definition to make sure that neither the first nor the last data point in the PDW is selected as the peak. The first data point would be the highest in case of a (negative) habituation drift while the last data point would be highest in case of an increasing drift - both of which would not indicate a stimulus induced SCR and hence were classified as a 'zero' or 'non-response' by Pineles. In contrast, the studies included in our systematic literature search did not employ such an iterative algorithm (despite many of them providing the study by Pineles as a reference for their approach) and hence did not include any zero or nonresponses. Our results show that the time-point of peak detection for the CS- is indeed often at the start of the PDW (i.e., first data point) which suggests that it indeed may capture the highest value of a habituating signal. It is particularly striking that this is the case for all PDW start values used. For the CS+ in turn, most peaks selected represent the value at the last data point of the PDW for all values of the PDW - also for those that cannot suffer from interference of the physiological US response (e.g., approach 4 for the $6 s$ CS durations). Possibly, this might reflect anticipatory responses, or second interval responses, that potentially may increase skin conductance values towards the end of the CS+ duration with increasing proximity to the anticipated US. Strikingly, US responses which are often large, clearly defined and are 
consistently elicited, are normally distributed in the US specific PDW with most responses peaking at about 4s. It should also be noted that we also observe a peak of peak detection at about 4s post CS onset for both the CS- and the CS+, which likely represent the true stimulus induced SCRs.

\subsubsection{Additional problems with BLC based response quantification: non-responder definition}

Different SCR response quantification approaches do not only lead to potentially different outcomes as outlined here, but also have potential implications for the application of commonly used postprocessing procedures. For instance, the identification of performance-based exclusion of SCR "nonlearner" (for a critical evaluation and discussion see Lonsdorf, Klingelhöfer-Jens, et al., 2019) may diverge between the approaches employed here: While SCR amplitudes quantified through the TTP approach cannot be smaller than zero, the BLC approach can yield negative values and we have demonstrated that this leads to differences in CS discrimination despite identical underlying raw data. Consequently, applying identical criteria for the definition of non-learning when using different SCR quantification approaches necessarily leads to different exclusion, which can be highly problematic (for a general criticism of this idea see Lonsdorf, Klingelhöfer-Jens, et al., 2019).

\subsubsection{Additional problems with SC quantification: Procedural challenges}

The systematic literature search revealed a number of difficulties in extracting and classifying the parameters used for SCR quantification. Specific difficulties included: a) unclear documentation of the used software program (e.g., Matlab, Acknowledge, custom-made) for either TTP scoring and/or BLC approaches b) insufficient descriptions of procedures and settings c) sometimes incorrect references used to refer reader to the procedure and d) unclear description of whether a time-window referred to the time-window in which the response onset (OLW) or the response peak (PDW) had to occur.

Commonly, absolute numerical values in seconds were reported for the time-windows used (such as start and end of PDW) without explicitly using a reference to the CS duration. These absolute numerical values without explicit reference to the experimental timing (i.e., CS duration, CS-US interval) render cross-study comparisons more difficult and may lead to problems when adopting a specific numeric time window identified in the literature to data generated from a study with different experimental timings. This may be particularly difficult for novices in the field or researchers outside the field that cannot be expected to infer that the criteria employed are only valid under the given experimental circumstances and need to be adjusted for the specific experimental timings. For example, the seemingly short PDW end at $4.5 \mathrm{~s}$ (approach 6 in Figure 1) in fact refers to $+0.5 \mathrm{~s}$ after CSoffset in that particular study. Thus, we strongly recommend to explicitly use a reference to the CS duration when reporting such time windows.

Furthermore, it would be helpful if the custom-made scripts used for response quantification would be made publicly available upon publication to facilitate an in-depth and unambiguous reproduction of procedures that counteracts that each team has to "re-invent the wheel" which may increases the risk of unintended and unnoticed differences between procedures.

\subsection{Summary and Outlook}

Taken together, our multiverse approach shows that a main effect of CS discrimination could be replicated independent of which of the 151 "ways", in other words exact SC quantification approaches was chosen. 
However, on a descriptive level, systematic patterns could be observed and might become more pronounced when effects with more subtle effect sizes are of interest.

Crucially, the stimulus specific disagreement observed between different categories of non-model based quantification approaches (i.e., BLC-SCL and TTP, and BLC-SCR and TTP) is concerning and underlines that even though effect sizes are comparable, this does not imply that there may not be systematic biases.

Of note, baseline correction methods are not confined to skin conductance research but are also applied to other physiological data such as EEG (Alday, 2019) and pupillometry (Mathôt et al., 2018). Interestingly, these approaches have also been criticized there in terms of their necessity. For pupillometric data, certain baseline corrections have been shown to be favored over others as they cause distortion of data, and the corrections can also reduce the sensitivity particularly to find weak effects (Mathôt et al., 2018).

It is indeed tempting to search for "the best" or "the optimal" approach to SCR quantification. It has been suggested that the best approach might be identified by the approach yielding the largest effect size. Yet, we showcase that merely using effect sizes as a criterion is insufficient. We have shown before (Kuhn et al., 2021) that the optimal approach depends on specific procedural details. Yet, given the specific problems of BLC based SCR quantification showcased here suggests that BLC based approaches may not be an optimal choice - at least when not adopting an iterative approach as in Pineles et al., (2009). Future studies using simulated data in which the ground truth is known and consequently allowing to test under what systematic processing conditions an effect is over- or under-estimated may be helpful to answer this question. 


\section{References}

Ahmed, O., \& Lovibond, P. F. (2019). Rule-based processes in generalisation and peak shift in human fear conditioning. Quarterly Journal of Experimental Psychology, 72(2), 118-131.

https://doi.org/10.1177/1747021818766461

Alday, P. M. (2019). How much baseline correction do we need in ERP research? Extended GLM model can replace baseline correction while lifting its limits. Psychophysiology, 56(12), e13451. https://doi.org/10.1111/psyp.13451

Bach, D. R., Flandin, G., Friston, K. J., \& Dolan, R. J. (2009). Time-series analysis for rapid event-related skin conductance responses. Journal of Neuroscience Methods, 184(2), 224-234. https://doi.org/10.1016/j.jneumeth.2009.08.005

Bach, D. R., \& Friston, K. J. (2013). Model-based analysis of skin conductance responses: Towards causal models in psychophysiology. Psychophysiology, 50(1), 15-22. https://doi.org/10.1111/j.14698986.2012.01483.x

Bach, D. R., Friston, K. J., \& Dolan, R. J. (2013). An improved algorithm for model-based analysis of evoked skin conductance responses. Biological Psychology, 94(3), 490-497. https://doi.org/10.1016/j.biopsycho.2013.09.010

Benedek, M., \& Kaernbach, C. (2010). A continuous measure of phasic electrodermal activity. Journal of Neuroscience Methods, 190(1), 80-91. https://doi.org/10.1016/j.jneumeth.2010.04.028

Boucsein, W., Fowles, D. C., Grimnes, S., Ben-Shakhar, G., Roth, W., T., Dawson, M., E., \& Filion, D., L. (2012). Publication recommendations for electrodermal measurements. Psychophysiology, 49(8), 1017-1034. https://doi.org/10.1111/j.1469-8986.2012.01384.x

Brunson, J. C. (2020). ggalluvial: Layered Grammar for Alluvial Plots. Journal of Open Source Software, 5(49), 2017. https://doi.org/10.21105/joss.02017

Geller, D. A., McGuire, J. F., Orr, S. P., Small, B. J., Murphy, T. K., Trainor, K., Porth, R., Wilhelm, S., \& Storch, E. A. (2019). Fear extinction learning as a predictor of response to cognitive behavioral 
therapy for pediatric obsessive compulsive disorder. Journal of Anxiety Disorders, 64, 1-8. https://doi.org/10.1016/j.janxdis.2019.02.005

Grings, W. (1974). Recording of electrodermal phenomena. In Methods in Physiological Psychology (S. 273-296). Academic Press.

Gruss, L. F., \& Keil, A. (2019). Sympathetic responding to unconditioned stimuli predicts subsequent threat expectancy, orienting, and visuocortical bias in human aversive Pavlovian conditioning. Biological Psychology, 140, 64-74. https://doi.org/10.1016/j.biopsycho.2018.11.009

Kuhn, M., Gerlicher, A., \& Lonsdorf, T. B. (2021). Navigating the manifold of skin conductance response quantification approaches [Preprint]. PsyArXiv. https://doi.org/10.31234/osf.io/9h2kd Kurayama, T., Matsuzawa, D., Hirano, Y., \& Shimizu, E. (2019). Insensitivity of auditory mismatch negativity to classical fear conditioning and extinction in healthy humans. 5.

LeBel, E. P., McCarthy, R. J., Earp, B. D., Elson, M., \& Vanpaemel, W. (2018). A Unified Framework to Quantify the Credibility of Scientific Findings. Advances in Methods and Practices in Psychological Science, 1(3), 389-402. https://doi.org/10.1177/2515245918787489

Lee, J. C., Hayes, B. K., \& Lovibond, P. F. (2018). Peak shift and rules in human generalization. Journal of Experimental Psychology: Learning, Memory, and Cognition, 44(12), 1955-1970. https://doi.org/10.1037/xIm0000558

Lee, J. C., Lovibond, P. F., Hayes, B. K., \& Navarro, D. J. (2019). Negative evidence and inductive reasoning in generalization of associative learning. Journal of Experimental Psychology: General, 148(2), 289-303. https://doi.org/10.1037/xge0000496

Lim, C. L., Rennie, C., Barry, R. J., Bahramali, H., Lazzaro, I., Manor, B., \& Gordon, E. (1997). Decomposing skin conductance into tonic and phasic components. International Journal of Psychophysiology, 25(2), 97-109. https://doi.org/10.1016/S0167-8760(96)00713-1 
Marin, M., Barbey, F., Rosenbaum, B. L., Hammoud, M. Z., Orr, S. P., \& Milad, M. R. (2020). Absence of conditioned responding in humans: A bad measure or individual differences? Psychophysiology, 57(1). https://doi.org/10.1111/psyp.13350

Mathôt, S., Fabius, J., Van Heusden, E., \& Van der Stigchel, S. (2018). Safe and sensible preprocessing and baseline correction of pupil-size data. Behavior Research Methods, 50(1), 94-106. https://doi.org/10.3758/s13428-017-1007-2

McGlade, A. L., Zbozinek, T. D., Treanor, M., \& Craske, M. G. (2019). Pilot for novel context generalization paradigm. Journal of Behavior Therapy and Experimental Psychiatry, 62, 49-56. https://doi.org/10.1016/j.jbtep.2018.08.009

Mertens, G., Leer, A., van Dis, E. A. M., Vermeer, L., Steenhuizen, A., van der Veen, L., \& Engelhard, I. M. (2019). Secondary extinction reduces reinstatement of threat expectancy and conditioned skin conductance responses in human fear conditioning. Journal of Behavior Therapy and Experimental Psychiatry, 62, 103-111. https://doi.org/10.1016/j.jbtep.2018.09.007

Morey, R. D., \& Rouder, J. N. (2018). BayesFactor: Computation of Bayes Factors for Common Designs. https://CRAN.R-project.org/package=BayesFactor

Ooms, J. (2021). magick: Advanced Graphics and Image-Processing in R. https://CRAN.Rproject.org/package=magick

Pedersen, T. L. (2020). patchwork: The Composer of Plots. https://CRAN.Rproject.org/package=patchwork

Pineles, S. L., Orr, M. R., \& Orr, S. P. (2009). An alternative scoring method for skin conductance responding in a differential fear conditioning paradigm with a long-duration conditioned stimulus. Psychophysiology, 46(5), 984-995. https://doi.org/10.1111/j.1469-8986.2009.00852.x

Rattel, J. A., Wegerer, M., Miedl, S. F., Blechert, J., Grünberger, L. M., Craske, M. G., \& Wilhelm, F. H. (2019). Peritraumatic unconditioned and conditioned responding explains sex differences in 
intrusions after analogue trauma. Behaviour Research and Therapy, 116, 19-29.

https://doi.org/10.1016/j.brat.2019.01.009

Scheveneels, S., Boddez, Y., De Ceulaer, T., \& Hermans, D. (2019). Ruining the surprise: The effect of safety information before extinction on return of fear. Journal of Behavior Therapy and Experimental Psychiatry, 63, 73-78. https://doi.org/10.1016/j.jbtep.2018.11.001

Simonsohn, U., Simmons, J. P., \& Nelson, L. D. (2020). Specification curve analysis. Nature Human Behaviour, 4(11), 1208-1214. https://doi.org/10.1038/s41562-020-0912-z

Vogel, S., Klumpers, F., Kroes, M. C. W., Oplaat, K. T., Krugers, H. J., Oitzl, M. S., Joëls, M., \& Fernández, G. (2015). A Stress-Induced Shift From Trace to Delay Conditioning Depends on the Mineralocorticoid Receptor. Biological Psychiatry, 78(12), 830-839. https://doi.org/10.1016/j.biopsych.2015.02.014

Wickham, H. (2016). ggplot2: Elegant Graphics for Data Analysis. Springer-Verlag New York. https://ggplot2.tidyverse.org

Yoshiike, T., Honma, M., Yamada, N., Kim, Y., \& Kuriyama, K. (2018). Effects of bright light exposure on human fear conditioning, extinction, and associated prefrontal activation. Physiology \& Behavior, 194, 268-276. https://doi.org/10.1016/j.physbeh.2018.06.015 


\section{Author notes}

Grant funding sources: This work was funded by grants from the German Research Foundation (DFG) to TBL (DFG LO1980/7-1 and CRC58; subproject B07, INST 211/633-1).

The authors declare no conflict of interest.

Contact details for reprints: Tina B. Lonsdorf, t.lonsdorf@uke.de 


\section{Supplementary Materials}

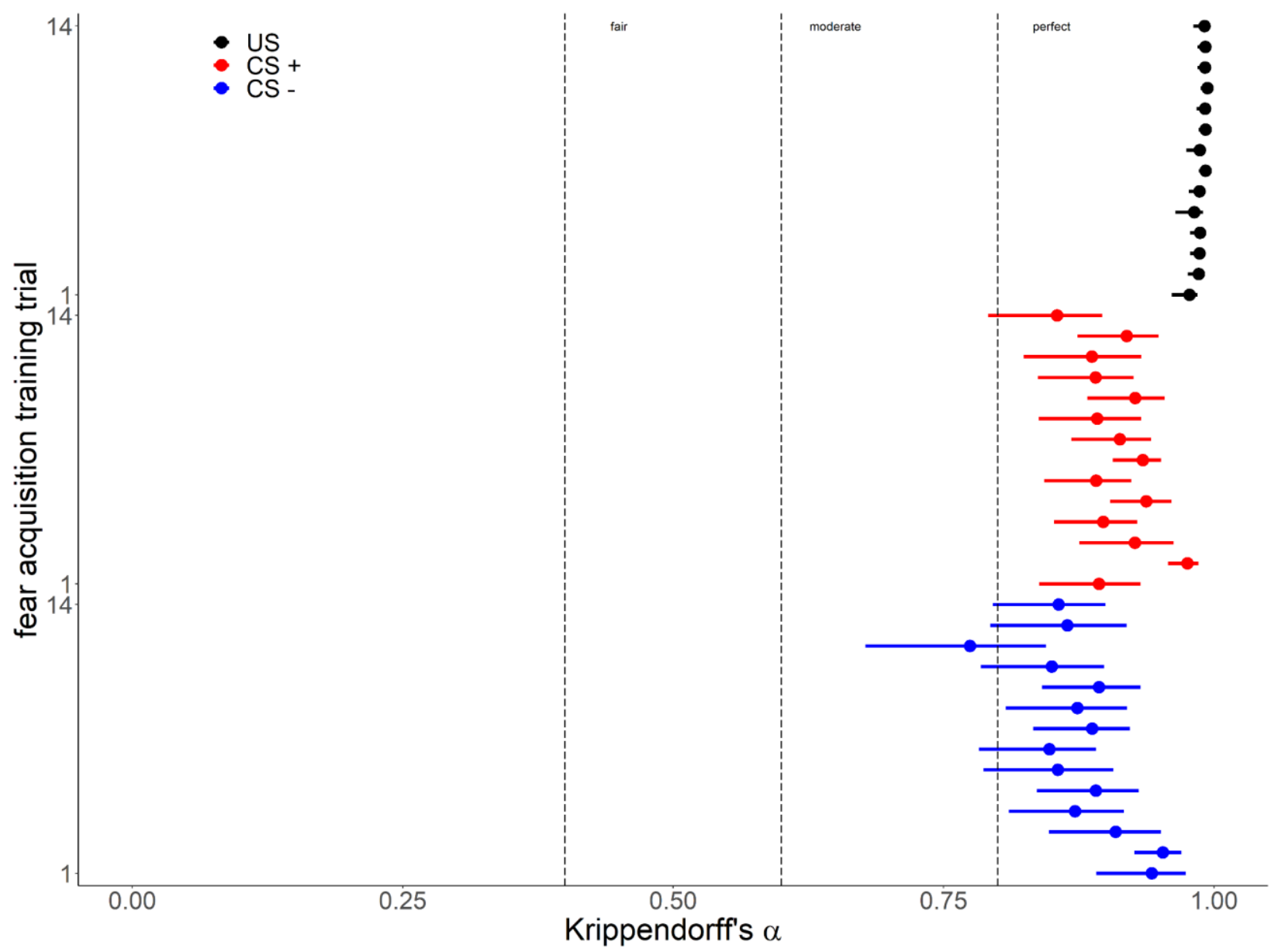

Supplementary Figure 1. Krippendorff's $\alpha$ values for SCR BLC approaches 1 to 8 , excluding SCL BLC9. 
Supplementary Table 1. Mean estimates of Krippendorff's $\alpha$ averaged per stimulus type (CS+, CS-, US) as well as minimum and maximum estimate per stimulus type for each of the pairwise comparisons of approaches. Approach X/Y refers to BLC 1-9, and TTP.

\begin{tabular}{|c|c|c|c|c|c|}
\hline approach X & approach Y & stimulus & mean & minimum & maximum \\
\hline 1 & 2 & CS- & 0,92 & 0,87 & 0,96 \\
\hline 1 & 2 & CS+ & 0,90 & 0,83 & 0,98 \\
\hline 1 & 2 & US & 0,99 & 0,97 & 1,00 \\
\hline 1 & 3 & CS- & 0,89 & 0,68 & 1,00 \\
\hline 1 & 3 & CS+ & 0,98 & 0,96 & 1,00 \\
\hline 1 & 3 & US & 1,00 & 1,00 & 1,00 \\
\hline 1 & 4 & CS- & 0,84 & 0,76 & 0,93 \\
\hline 1 & 4 & CS+ & 0,94 & 0,91 & 0,97 \\
\hline 1 & 4 & US & 0,98 & 0,97 & 0,99 \\
\hline 1 & 5 & CS- & 0,90 & 0,86 & 0,95 \\
\hline 1 & 5 & CS+ & 0,80 & 0,65 & 0,95 \\
\hline 1 & 5 & US & 0,98 & 0,96 & 0,99 \\
\hline 1 & 6 & CS- & 0,94 & 0,84 & 0,98 \\
\hline 1 & 6 & CS+ & 0,97 & 0,96 & 0,99 \\
\hline 1 & 6 & US & 0,99 & 0,98 & 0,99 \\
\hline 1 & 7 & CS- & 0,89 & 0,76 & 0,96 \\
\hline 1 & 7 & CS+ & 0,94 & 0,91 & 0,97 \\
\hline 1 & 7 & US & 0,99 & 0,98 & 0,99 \\
\hline 1 & 8 & CS- & 0,87 & 0,75 & 0,94 \\
\hline 1 & 8 & CS+ & 0,89 & 0,81 & 0,96 \\
\hline 1 & 8 & US & 0,98 & 0,97 & 0,99 \\
\hline 1 & 9 & CS- & 0,52 & 0,31 & 0,67 \\
\hline 1 & 9 & CS+ & 0,84 & 0,77 & 0,92 \\
\hline 1 & 9 & US & 0,86 & 0,75 & 0,97 \\
\hline 1 & TTP & CS- & 0,54 & 0,24 & 0,69 \\
\hline
\end{tabular}




\begin{tabular}{|c|c|c|c|c|c|}
\hline 1 & TTP & CS+ & 0,83 & 0,65 & 0,90 \\
\hline 1 & TTP & US & 0,84 & 0,77 & 0,89 \\
\hline 2 & 3 & CS- & 0,80 & 0,56 & 0,96 \\
\hline 2 & 3 & CS+ & 0,89 & 0,81 & 0,98 \\
\hline 2 & 3 & US & 0,99 & 0,97 & 1,00 \\
\hline 2 & 4 & CS- & 0,92 & 0,87 & 0,99 \\
\hline 2 & 4 & $\mathrm{CS}+$ & 0,95 & 0,91 & 0,98 \\
\hline 2 & 4 & US & 0,98 & 0,97 & 0,99 \\
\hline 2 & 5 & CS- & 0,93 & 0,85 & 0,97 \\
\hline 2 & 5 & $\mathrm{CS}+$ & 0,92 & 0,70 & 0,98 \\
\hline 2 & 5 & US & 0,99 & 0,98 & 0,99 \\
\hline 2 & 6 & CS- & 0,86 & 0,76 & 0,92 \\
\hline 2 & 6 & CS + & 0,85 & 0,77 & 0,95 \\
\hline 2 & 6 & US & 0,98 & 0,93 & 0,99 \\
\hline 2 & 7 & |CS- & 0,84 & 0,64 & 0,96 \\
\hline 2 & 7 & CS+ & 0,94 & 0,91 & 0,98 \\
\hline 2 & 7 & US & 0,99 & 0,98 & 0,99 \\
\hline 2 & 8 & CS- & 0,85 & 0,65 & 0,97 \\
\hline 2 & 8 & $\mathrm{CS}+$ & 0,97 & 0,93 & 0,99 \\
\hline 2 & 8 & US & 0,99 & 0,98 & 1,00 \\
\hline 2 & 9 & CS- & 0,55 & 0,40 & 0,72 \\
\hline 2 & 9 & CS+ & 0,81 & 0,67 & 0,93 \\
\hline 2 & 9 & US & 0,86 & 0,76 & 0,98 \\
\hline 2 & TTP & CS- & 0,53 & 0,25 & 0,68 \\
\hline 2 & TTP & CS+ & 0,78 & 0,66 & 0,84 \\
\hline 2 & TTP & US & 0,83 & 0,76 & 0,88 \\
\hline 3 & 4 & CS- & 0,71 & 0,46 & 0,92 \\
\hline 3 & 4 & CS+ & 0,92 & 0,87 & 0,97 \\
\hline
\end{tabular}




\begin{tabular}{|c|c|c|c|c|c|}
\hline 3 & 4 & US & 0,98 & 0,97 & 0,99 \\
\hline 3 & 5 & CS- & 0,83 & 0,62 & 0,93 \\
\hline 3 & 5 & CS+ & 0,80 & 0,67 & 0,96 \\
\hline 3 & 5 & US & 0,98 & 0,96 & 0,99 \\
\hline 3 & 6 & CS- & 0,88 & 0,72 & 0,98 \\
\hline 3 & 6 & CS+ & 0,96 & 0,93 & 0,99 \\
\hline 3 & 6 & US & 0,99 & 0,98 & 0,99 \\
\hline 3 & 7 & CS- & 0,91 & 0,82 & 0,96 \\
\hline 3 & 7 & CS+ & 0,95 & 0,92 & 0,98 \\
\hline 3 & 7 & US & 0,99 & 0,98 & 0,99 \\
\hline 3 & 8 & CS- & 0,90 & 0,81 & 0,95 \\
\hline 3 & 8 & CS+ & 0,90 & 0,84 & 0,97 \\
\hline 3 & 8 & US & 0,98 & 0,97 & 0,99 \\
\hline 3 & 9 & CS- & 0,45 & 0,21 & 0,64 \\
\hline 3 & 9 & CS+ & 0,83 & 0,75 & 0,93 \\
\hline 3 & 9 & US & 0,86 & 0,75 & 0,97 \\
\hline 3 & TTP & |CS- & 0,55 & 0,32 & 0,71 \\
\hline 3 & TTP & CS+ & 0,82 & 0,60 & 0,89 \\
\hline 3 & TTP & US & 0,84 & 0,76 & 0,89 \\
\hline 4 & 5 & CS- & 0,86 & 0,79 & 0,96 \\
\hline 4 & 5 & CS+ & 0,85 & 0,68 & 0,98 \\
\hline 4 & 5 & US & 0,99 & 0,97 & 1,00 \\
\hline 4 & 6 & CS- & 0,83 & 0,69 & 0,92 \\
\hline 4 & 6 & CS+ & 0,91 & 0,85 & 0,98 \\
\hline 4 & 6 & US & 0,98 & 0,96 & 0,99 \\
\hline 4 & 7 & CS- & 0,79 & 0,56 & 0,96 \\
\hline 4 & 7 & CS+ & 0,97 & 0,93 & 1,00 \\
\hline 4 & 7 & US & 0,99 & 0,98 & 1,00 \\
\hline
\end{tabular}




\begin{tabular}{|c|c|c|c|c|c|}
\hline 4 & 8 & CS- & 0,78 & 0,56 & 0,95 \\
\hline 4 & 8 & $\mathrm{CS}+$ & 0,94 & 0,89 & 0,99 \\
\hline 4 & 8 & US & 0,99 & 0,98 & 1,00 \\
\hline 4 & 9 & CS- & 0,66 & 0,54 & 0,79 \\
\hline 4 & 9 & CS+ & 0,88 & 0,76 & 0,94 \\
\hline 4 & 9 & US & 0,87 & 0,73 & 0,99 \\
\hline 4 & TTP & CS- & 0,48 & 0,14 & 0,72 \\
\hline 4 & TTP & CS+ & 0,80 & 0,68 & 0,87 \\
\hline 4 & TTP & US & 0,82 & 0,77 & 0,87 \\
\hline 5 & 6 & CS- & 0,91 & 0,87 & 0,95 \\
\hline 5 & 6 & CS+ & 0,76 & 0,61 & 0,95 \\
\hline 5 & 6 & US & 0,99 & 0,93 & 1,00 \\
\hline 5 & 7 & CS- & 0,91 & 0,80 & 0,98 \\
\hline 5 & 7 & CS+ & 0,87 & 0,75 & 0,99 \\
\hline 5 & 7 & US & 1,00 & 0,99 & 1,00 \\
\hline 5 & 8 & CS- & 0,93 & 0,80 & 0,99 \\
\hline 5 & 8 & CS + & 0,93 & 0,77 & 0,99 \\
\hline 5 & 8 & US & 1,00 & 1,00 & 1,00 \\
\hline 5 & 9 & CS- & 0,56 & 0,35 & 0,70 \\
\hline 5 & 9 & CS + & 0,73 & 0,61 & 0,93 \\
\hline 5 & 9 & US & 0,89 & 0,79 & 0,98 \\
\hline 5 & TTP & CS- & 0,50 & 0,20 & 0,65 \\
\hline 5 & TTP & CS+ & 0,70 & 0,50 & 0,79 \\
\hline 5 & TTP & US & 0,82 & 0,76 & 0,86 \\
\hline 6 & 7 & CS- & 0,95 & 0,90 & 0,97 \\
\hline 6 & 7 & CS+ & 0,93 & 0,88 & 0,98 \\
\hline 6 & 7 & US & 0,99 & 0,96 & 1,00 \\
\hline 6 & 8 & CS- & 0,92 & 0,85 & 0,96 \\
\hline
\end{tabular}




\begin{tabular}{|c|c|c|c|c|c|}
\hline 6 & 8 & CS+ & 0,87 & 0,79 & 0,97 \\
\hline 6 & 8 & US & 0,99 & 0,94 & 1,00 \\
\hline 6 & 9 & CS- & 0,58 & 0,36 & 0,68 \\
\hline 6 & 9 & CS+ & 0,85 & 0,79 & 0,93 \\
\hline 6 & 9 & US & 0,88 & 0,78 & 0,94 \\
\hline 6 & TTP & CS- & 0,49 & 0,19 & 0,66 \\
\hline 6 & TTP & CS+ & 0,80 & 0,62 & 0,89 \\
\hline 6 & TTP & US & 0,82 & 0,75 & 0,90 \\
\hline 7 & 8 & CS- & 0,98 & 0,96 & 1,00 \\
\hline 7 & 8 & CS+ & 0,97 & 0,93 & 0,99 \\
\hline 7 & 8 & US & 1,00 & 1,00 & 1,00 \\
\hline 7 & 9 & CS- & 0,54 & 0,29 & 0,71 \\
\hline 7 & 9 & CS+ & 0,87 & 0,77 & 0,94 \\
\hline 7 & 9 & US & 0,89 & 0,78 & 0,99 \\
\hline 7 & TTP & CS- & 0,51 & 0,21 & 0,68 \\
\hline 7 & TTP & CS+ & 0,79 & 0,62 & 0,87 \\
\hline 7 & TTP & US & 0,82 & 0,76 & 0,86 \\
\hline 8 & 9 & CS- & 0,52 & 0,28 & 0,70 \\
\hline 8 & 9 & CS+ & 0,82 & 0,68 & 0,94 \\
\hline 8 & 9 & US & 0,89 & 0,78 & 0,98 \\
\hline 8 & TTP & CS- & 0,51 & 0,23 & 0,66 \\
\hline 8 & TTP & CS+ & 0,76 & 0,63 & 0,84 \\
\hline 8 & TTP & US & 0,82 & 0,76 & 0,86 \\
\hline 9 & TTP & CS- & 0,22 & $-0,11$ & 0,49 \\
\hline 9 & TTP & CS+ & 0,71 & 0,53 & 0,79 \\
\hline 9 & TTP & US & 0,71 & 0,60 & 0,84 \\
\hline
\end{tabular}


Insert link to suppfig_2_curve_BF.gif here.

Supplementary figure 2. Color coded animated version of the specification curve of effect sizes resulting from main effects of CS discrimination within all 150 BLC approaches and 1 TTP approach. Color coding by each of the four parameters (BLW = baseline window, PDW-start = peak detection window start, PDW-end $=$ peak detection window end, function = aggregate function in peak detection window). 